arXiv: math.ST: 1503.03212

\title{
Multivariate Generalized Gram-Charlier Series in Vector Notations
}

\author{
Dharmani Bhaveshkumar C. \\ Dhirubhai Ambani Institute of \\ Information Es Communication Technology (DAIICT), \\ Gandhinagar, Gujarat, INDIA - 382001 e-mail: dharmanibc@gmail.com
}

\begin{abstract}
The article derives multivariate Generalized Gram-Charlier (GGC) series that expands an unknown joint probability density function $(p d f)$ of a random vector in terms of the differentiations of the joint $p d f$ of a reference random vector. Conventionally, the higher order differentiations of a multivariate $p d f$ in GGC series will require multi-element array or tensor representations. But, the current article derives the GGC series in vector notations. The required higher order differentiations of a multivariate $p d f$ in vector notations are achieved through application of a specific Kronecker product based differentiation operator. Overall, the article uses only elementary calculus of several variables; instead Tensor calculus; to achieve the extension of an existing specific derivation for GGC series in univariate to multivariate. The derived multivariate GGC expression is more elementary as using vector notations compare to the coordinatewise tensor notations and more comprehensive as apparently more nearer to its counterpart for univariate. The same advantages are shared by the other expressions obtained in the article; such as the mutual relations between cumulants and moments of a random vector, integral form of a multivariate $p d f$, integral form of the multivariate Hermite polynomials, the multivariate Gram-Charlier A (GCA) series and others.
\end{abstract}

Primary 62E17, 62H10; Secondary 60E10.

Keywords and phrases: Multivariate Generalized Gram-Charlier (GGC) series; Multivariate Gram-Charlier A (GCA) series; Multivariate Vector Hermite Polynomials; Kronecker Product; Vector moments; Vector cumulants.

\section{Introduction}

The Generalized Gram-Charlier (GGC) series expands an unknown pdf as a linear combination of the increasing order differentiations of a reference $p d f$, where the coefficients of expansion involve cumulant differences between those of an unknown $p d f$ and a reference $p d f$. The GGC expansions are used to approximate $p d f$ and functions of $p d f$ in Statistics, Machine Learning, Economics, Chemistry, Astronomy and other application areas. There have been used Poisson's distribution (Aroian, 1937), log-normal distribution (Aroian, 1947), binomial distribution (Rietz, 1927), gamma distribution (Bowers, 1966) and others as reference $p d f \mathrm{~s}$. But, the Gram-Charlier (GC) expansion with Gaussian density as a reference $p d f$ is the most popular and identified as the GramCharlier A (GCA) series. Specifically, the GCA series is used for test of Gaussianity or near Gaussian pdf approximations (Aroian, 1947; Viehland, 1994;

1

imsart-generic ver. 2011/11/15 file: arXivndGramC.tex date: January 27, 2016 
Girolami and Fyfe, 1996; Jondeau and Rockinger, 2001; Del Brio et al., 2009), for entropy measure and independence measure approximations (Amari et al., 1996; Hyvärinen et al., 2001), for optima analysis through derivatives of $p d f$ (Boscolo and Roychowdhury, 2003), for time-frequency analysis (Cohen, 1998) and others. The rearrangement of the terms in GCA series results into the Edgeworth series with better convergence property.

There exists multiple ways to derive univariate GCA series, as reported by Hald (2000); Hald and Steffensen (2002). It's generalization to univariate GGC series is derived by Schleher (1977); Cohen (1998); Berberan-Santos (2007); Cohen (2011).

The multivariate GGC or GCA series derivation requires multivariate representations of the Taylor series, the increasing order differentiations of a reference $p d f$ and the cumulants. To signify the representation issue in required multivariate extensions; it is worth quoting Terdik (2002) that says, ' . . though the generalizations to higher dimensions may be considered straightforward, the methodology and the mathematical notations get quite cumbersome when dealing with the higher order derivatives of the characteristic function or other functions of a random vector...'?

Conventionally, the higher order differentiations of a multivariate $p d f$; and therefore, the multivariate cumulants and multivariate Hermite polynomials; require multilinear representations. It is known and acknowledged historically that going from matrix like notations to tensor notations for multivariate cumulants and multivariate Hermite polynomials have made the representation more transparent and the calculations more simpler (McCullagh, 1987). Though the tensor notations have advantages over the matrix notations, they require componentwise separate representations and are more tedious compare to the vector notations. As a unified and comprehensive solution to this, there has been used an approach based only on elementary calculus of several variables by Terdik (2002). The approach uses a specific Kronecker product based differential operator, identified as the 'K-derivative operator', to achieve vectorization of the Jacobian matrix of a multivariate vector function. The successive applications of this K-derivative operator achieves vectorization of the higher order derivatives also. Using this approach, there have been derived the multivariate Taylor series and the higher order cumulants (Terdik, 2002; S. Rao Jammalamadaka and Terdik, May, 2006); as well, the multivariate Hermite polynomials (Terdik, 2002) in vector notations resulting into more transparent representations. In fact, it could be noticed that the same approach has been first used to derive vector Hermite polynomials by Holmquist (1996); and later ${ }^{1}$ it has been formalized and generalized by Terdik (2002).

There exists various approaches deriving multivariate GCA series with various representations. They include GCA series representation using multielement matrix notations for moments and cumulants by Sauer and Heydt (1979); using recursive formula for Hermite polynomials by Berkowitz and Garner (1970); using tensor representation for cumulants and Hermite polynomials by McCullagh

${ }^{1}$ Somehow, the citation for (Holmquist, 1996) is not found in article (Terdik, 2002). 
(1987, Chapter 5); using tensor representation for cumulants and involving multivariate Bell polynomials by Withers and Nadarajah (2014); using vector moments and vector Hermite polynomials by Holmquist (1996) and others. There exists various derivations for multivariate Edgeworth series (Davis, Dec., 1976; Skovgaard, 1986; Amari and Kumon, 1983; McCullagh, 1987; Kollo and von Rosen, 1998; Withers and Nadarajah, 2014). There also exists multivariate GGC series, derived by McCullagh (1987, Chapter 5), in tensor notations. But, as per the author's knowledge, there exists neither the multivariate GGC series nor the multivariate Edgeworth series in vector notations. For the ease of the readers in following and comparing the various representations; the existing representations of multivariate GGC series and multivariate GCA series are shown and compared in A.

Overall, to take advantages due to the recent advancement in representation, this article extends a specific derivation for univariate GGC series by Berberan-Santos (2007) to multivariate; using only elementary calculus of several variables instead of Tensor calculus. As a by product, it also derives mutual relations between vector cumulants and vector moments of a random vector; integral form of the multivariate $p d f$; integral form of the multivariate vector Hermite polynomials and the multivariate GCA series. All the derived multivariate expressions are more elementary as using vector notations and more comprehensive as apparently more nearer to their counterparts for univariate; compare to their coordinatewise tensor notations. The intermediate theoretical results, in the article, are verified using suitable known examples.

Towards the aim of the article, the next Section 2 briefs some necessary background on the Kronecker product and a way to obtain vectorization of the higher order differentiations of a multivariate $p d f$. It also obtains the required multivariate Taylor series expansion using the derived notations. After the preliminary background, this article follows almost the same sequence for multivariate as that in (Berberan-Santos, 2007) for univariate. The Section 3 uses the characteristic function and the generating functions to derive cumulants and moments of a random vector in vector notations with their mutual relationships. The Section 4 obtains multivariate $p d f$ in terms of its vector cumulants. The expressions for derivatives of multivariate Gaussian density and vector Hermite polynomials are derived in Section 5. The Section 6 derives multivariate GCA series by representing an unknown $p d f$ in terms of the Gaussian $p d f$ as a reference. The Section 7 derives GGC Expansion, representing an unknown $p d f$ in terms of the a known reference $p d f$. The Section 8 derives an unknown characteristic function of a random vector in terms of a reference characteristic function. The Section 9 derives the same GGC expansion in a more compact way that summarizes the approach of the whole derivation. Finally, Section 10 concludes the article. For the sake of clarity; the calculation details, the proofs and the expressions for existing multivariate expansions are kept in appendix at the end of the article. 


\section{Vectorization of the higher order differentiations}

The section briefs the Kronecker product and the way it can be applied to achieve vectorization of the higher order differentiations of a multivariate $p d f$. Based on it, the multivariate Taylor series is obtained in vector notations. More details can be found on the Kronecker Product in (Magnus and Neudecker, 1999, Chapter 2), on achieving vectorization of the higher order differentiations in (Terdik, 2002; S. Rao Jammalamadaka and Terdik, May, 2006) and on the commutation matrices in (Magnus and Neudecker, 1999, Chapter 3, Section 7).

Definition 1 (Kronecker Product Operator $(\otimes)$ ). The Kronecker Product Operator $(\otimes)$ between matrices $\mathbf{A}$ with size $p \times q$ and $\mathbf{B}$ with size $m \times n$ is defined as:

$$
\mathbf{A} \otimes \mathbf{B}=\left[\begin{array}{cccc}
a_{11} \mathbf{B} & a_{12} \mathbf{B} & \cdots & a_{1 q} \mathbf{B} \\
a_{21} \mathbf{B} & a_{22} \mathbf{B} & \cdots & a_{2 q} \mathbf{B} \\
\vdots & \vdots & \ddots & \vdots \\
a_{p 1} \mathbf{B} & a_{p 2} \mathbf{B} & \cdots & a_{p q} \mathbf{B}
\end{array}\right]
$$

The resultant matrix is of dimension $p m \times q n$. As a further example; let $\mathbf{A}$ is with size $p \times 1$ and $\mathbf{B}$ is with size $m \times 1$, then $\mathbf{A} \otimes \mathbf{B}^{\prime}$ is ${ }^{2}$ a matrix with size $p \times m . \mathbf{A} \otimes \mathbf{A}$ is symbolically represented as $\mathbf{A}^{\otimes 2}$ and has size $p^{2} \times 1$. In general, $\mathbf{A} \otimes \mathbf{A} \otimes \ldots \otimes \mathbf{A}$ (n times) is symbolically represented as $\mathbf{A}^{\otimes n}$ and has size $p^{n} \times 1$.

Definition 2 (Jacobian Matrix). Let $\boldsymbol{\lambda}=\left(\lambda_{1}, \lambda_{2}, \ldots, \lambda_{d}\right)^{\prime}, \boldsymbol{\lambda} \in \mathbb{R}^{d}$ and $\mathbf{f}(\boldsymbol{\lambda})=$ $\left(f_{1}(\lambda), f_{2}(\lambda), \ldots, f_{m}(\lambda)\right)^{\prime} \in \mathbb{R}^{m}$ be a differentiable $m$-component vector function. Then, Jacobian matrix of $\mathbf{f}(\boldsymbol{\lambda})(\mathbf{J}(\mathbf{f}))$ is an $m \times d$ matrix defined as under:

$$
\mathbf{J}(\mathbf{f}(\boldsymbol{\lambda}))=\frac{d \mathbf{f}}{d \boldsymbol{\lambda}}=\left[\frac{\partial \mathbf{f}}{\partial \lambda_{1}}, \frac{\partial \mathbf{f}}{\partial \lambda_{2}}, \ldots, \frac{\partial \mathbf{f}}{\partial \lambda_{d}}\right]=\left[\begin{array}{cccc}
\frac{\partial f_{1}}{\partial \lambda_{1}} & \frac{\partial f_{1}}{\partial \lambda_{2}} & \cdots & \frac{\partial f_{1}}{\partial \lambda_{d}} \\
\frac{\partial f_{2}}{\partial \lambda_{1}} & \ddots & & \vdots \\
\vdots & & \ddots & \vdots \\
\frac{\partial f_{m}}{\partial \lambda_{1}} & \frac{\partial f_{m}}{\partial \lambda_{2}} & \cdots & \frac{\partial f_{m}}{\partial \lambda_{d}}
\end{array}\right]
$$

Let the vector differential operator be defined as a column vector $\mathbf{D}_{\boldsymbol{\lambda}}=$ $\left(\frac{\partial}{\partial \lambda_{1}}, \frac{\partial}{\partial \lambda_{2}}, \ldots, \frac{\partial}{\partial \lambda_{d}}\right)^{\prime}$, then the Jacobian matrix, in terms of the $\mathbf{D}_{\boldsymbol{\lambda}}$, can be re-written as:

$\mathbf{J}(\mathbf{f}(\boldsymbol{\lambda}))=\mathbf{D}_{\boldsymbol{\lambda}}(\mathbf{f})=\mathbf{f}(\boldsymbol{\lambda}) \mathbf{D}_{\boldsymbol{\lambda}}^{\prime}=\left(f_{1}(\boldsymbol{\lambda}), f_{2}(\boldsymbol{\lambda}), \ldots, f_{m}(\boldsymbol{\lambda})\right)^{\prime}\left(\frac{\partial}{\partial \lambda_{1}}, \frac{\partial}{\partial \lambda_{2}}, \ldots, \frac{\partial}{\partial \lambda_{d}}\right)$

This implies that to match the definition of differentiation from matrix calculus, the vector differential operator should be applied from the right to the left. This

\footnotetext{
${ }^{2}$ The symbol ' stands for Transpose of a matrix
} 
is same as the requirement to be satisfied on generalization of vector derivative to matrix derivative as discussed by Magnus (2010). So, applying vector derivative operator from right to the left, has been kept as a rule throughout the article.

Definition 3 (The K-derivative Operator). Let $\boldsymbol{\lambda}=\left(\lambda_{1}, \lambda_{2}, \ldots, \lambda_{d}\right)^{\prime}, \boldsymbol{\lambda} \in \mathbb{R}^{d}$; the vector differential operator $\mathbf{D}_{\boldsymbol{\lambda}}=\left(\frac{\partial}{\partial \lambda_{1}}, \frac{\partial}{\partial \lambda_{2}}, \ldots, \frac{\partial}{\partial \lambda_{d}}\right)^{\prime}$ and a differentiable m-component vector function $\mathbf{f}(\boldsymbol{\lambda})=\left(f_{1}(\lambda), f_{2}(\lambda), \ldots, f_{m}(\lambda)\right)^{\prime} \in \mathbb{R}^{m}$. Then, the Kronecker product between $\mathbf{D}_{\boldsymbol{\lambda}}$ and $\mathbf{f}(\boldsymbol{\lambda})$ is given as under:

$$
\begin{aligned}
& \mathbf{D}_{\boldsymbol{\lambda}}^{\otimes} \mathbf{f}(\boldsymbol{\lambda})=\left[\begin{array}{c}
f_{1}(\boldsymbol{\lambda}) \\
f_{2}(\boldsymbol{\lambda}) \\
\vdots \\
f_{m}(\boldsymbol{\lambda})
\end{array}\right] \otimes\left[\begin{array}{c}
\frac{\partial}{\partial \lambda_{1}} \\
\frac{\partial}{\partial \lambda_{2}} \\
\vdots \\
\frac{\partial}{\partial \lambda_{d}}
\end{array}\right]=V e c\left[\begin{array}{cccc}
\frac{\partial f_{1}}{\partial \lambda_{1}} & \frac{\partial f_{1}}{\partial \lambda_{2}} & \cdots & \frac{\partial f_{1}}{\partial \lambda_{d}} \\
\frac{\partial f_{2}}{\partial \lambda_{1}} & \ddots & & \vdots \\
\vdots & & \ddots & \vdots \\
\frac{\partial f_{m}}{\partial \lambda_{1}} & \frac{\partial f_{m}}{\partial \lambda_{2}} & \cdots & \frac{\partial f_{m}}{\partial \lambda_{d}}
\end{array}\right]^{\prime} \\
\Rightarrow \mathbf{D}_{\lambda}^{\otimes} \mathbf{f}(\boldsymbol{\lambda}) & =V e c\left(\frac{\partial \mathbf{f}}{\partial \boldsymbol{\lambda}^{\prime}}\right)^{\prime}=V e c\left(\frac{\partial}{\partial \boldsymbol{\lambda}} \mathbf{f}^{\prime}\right)
\end{aligned}
$$

where, the Vec operator converts $m \times d$ matrix into an $m d \times 1$ column vector by stacking the columns one after an other. The operator $\mathbf{D}_{\lambda}^{\otimes}$ is called Kronecker derivative operator or simply, K-derivative operator.

Thus, the Kronecker product with the vector differential operator, obtains vectorization of the transposed Jacobian of a vector function. Corresponding to the definition, the $k^{t h}$ order differentiation is given by:

$$
\mathbf{D}_{\lambda}^{\otimes k} \mathbf{f}=\mathbf{D}_{\lambda}^{\otimes}\left(\mathbf{D}_{\lambda}^{\otimes k-1} \mathbf{f}\right)=\left[f_{1}(\lambda), f_{2}(\lambda), \ldots, f_{m}(\lambda)\right]^{\prime} \otimes\left[\frac{\partial}{\partial \lambda_{1}}, \frac{\partial}{\partial \lambda_{2}}, \cdots, \frac{\partial}{\partial \lambda_{d}}\right]^{\prime \otimes k}
$$

The $\mathbf{D}_{\lambda}^{\otimes k} \mathbf{f}$ is a column vector of dimension $m d^{k} \times 1$. Some important properties of the K-derivative operator, those are useful in the further derivations, are listed in Appendix B.

\subsection{Application of the K-derivative operator to the multivariate Taylor series}

Let $\mathbf{x}=\left(X_{1}, X_{2}, \ldots, X_{d}\right)^{\prime}$ be a d-dimensional column vector and $f(\mathbf{x})$ be the function of several variables differentiable in each variable. Using the defined K-derivative operator, the Taylor series for $f(\mathbf{x})$, expanding it at origin, is given as:

$$
f(\mathbf{x})=\sum_{m=0}^{m=\infty} \frac{1}{m !} \mathbf{c}(m, d)^{\prime} \mathbf{x}^{\otimes m}
$$

where, $\mathbf{c}(m, d)$ is the vector of dimension $d^{m} \times 1$ and given in terms of the derivative vector $\mathbf{D}_{\mathbf{x}}=\left(\frac{\partial}{\partial x_{1}}, \frac{\partial}{\partial x_{2}}, \cdots, \frac{\partial}{\partial x_{d}}\right)^{\prime}$ as

$$
\mathbf{c}(m, d)=\left.\left(\mathbf{D}_{\mathbf{x}}^{\otimes m} f(\mathbf{x})\right)\right|_{\mathbf{x}=\mathbf{0}}
$$


The Taylor series expansion near $\boldsymbol{\lambda}=\mathbf{0}$, called the Maclaurian series, of some required functions based on the Equation (7) are derived in appendix C.

\section{Moments, cumulants and characteristic function of a random vector}

Let $\mathbf{x}=\left(X_{1}, X_{2}, \ldots, X_{d}\right)^{\prime}$ be a d-dimensional random vector and $f(\mathbf{x})$ be its joint $p d f$ differentiable in each variable.

The Characteristic function $(\mathcal{F})$ of $\mathbf{x}$ is defined as the expected value of $\mathrm{e}^{i \mathbf{x}^{\prime} \boldsymbol{\lambda}}$, where $\boldsymbol{\lambda}=\left(\lambda_{1}, \lambda_{2}, \ldots, \lambda_{d}\right)^{\prime}, \boldsymbol{\lambda} \in \mathbb{R}^{d}$. Also, both the characteristic function and the pdf are the Fourier Transform (F) of each other, in the sense they are dual.

$$
\mathcal{F}_{\mathbf{x}}(\boldsymbol{\lambda})=E\left\{\mathrm{e}^{i\left(\mathbf{x}^{\prime} \boldsymbol{\lambda}\right)}\right\}=\mathrm{F}(f(\mathbf{x}))=\int_{-\infty}^{\infty} \cdots \int_{-\infty}^{\infty} f(\mathbf{x}) \mathrm{e}^{i\left(\mathbf{x}^{\prime} \boldsymbol{\lambda}\right)} d \mathbf{x}
$$

Expanding $\mathrm{e}^{i \mathbf{x}^{\prime} \boldsymbol{\lambda}}$ using its Maclaurian series in Equation (80) in appendix C, we get:

$$
\mathcal{F}_{\mathbf{x}}(\boldsymbol{\lambda})=\sum_{k=0}^{\infty} \mathbf{m}(k, d)^{\prime} \frac{(i \boldsymbol{\lambda})^{\otimes k}}{k !}
$$

where, $\mathbf{m}(k, d)$ is the $k^{t h}$ order moment vector of dimension $d^{k} \times 1$ and given by

$$
\begin{aligned}
\mathbf{m}(k, d) & =\int_{\mathbb{R}^{d}} \mathbf{x}^{\otimes k} f(\mathbf{x}) d \mathbf{x} \\
\text { Also, } f(\mathbf{x})=\mathrm{F}^{-1}(\mathcal{F}(\lambda)) & =\mathrm{F}^{-1}\left(\sum_{k=0}^{\infty} \mathbf{m}(k, d)^{\prime} \frac{(i \boldsymbol{\lambda})^{\otimes k}}{k !}\right) \\
& =\sum_{k=0}^{\infty} \frac{\mathbf{m}(k, d)^{\prime}}{k !}\left(\frac{1}{(2 \pi)^{d}} \int_{\mathbb{R}^{d}}(i \boldsymbol{\lambda})^{\otimes k} \mathrm{e}^{-i \mathbf{x}^{\prime} \boldsymbol{\lambda}} d \boldsymbol{\lambda}\right) \\
& =\sum_{k=0}^{\infty}(-1)^{k} \frac{\mathbf{m}(k, d)^{\prime}}{k !} \mathbf{D}^{(k)} \delta(\mathbf{x})
\end{aligned}
$$

$(\because$ Proof in Appendix D.1)

The Moment Generating Function (MGF) of $f(\mathbf{X})$ is given as

$$
\begin{aligned}
\mathbf{M}(\boldsymbol{\lambda}) & =E\left\{\mathrm{e}^{\mathbf{x}^{\prime} \boldsymbol{\lambda}}\right\}=\int_{\mathbb{R}^{d}} f(\mathbf{X}) \mathrm{e}^{\mathbf{x}^{\prime} \boldsymbol{\lambda}} d \mathbf{X} \\
& =\sum_{k=0}^{\infty} \mathbf{m}(k, d)^{\prime} \frac{\boldsymbol{\lambda}^{\otimes k}}{k !} \quad\left(\because \text { Expanding } \mathrm{e}^{\mathbf{x}^{\prime} \boldsymbol{\lambda}}\right)
\end{aligned}
$$

Assuming $\mathbf{M}(\boldsymbol{\lambda})$ and $\mathcal{F}(\boldsymbol{\lambda})$ are expanded using Taylor series,

$$
\mathbf{m}(k, d)=\left.\mathbf{D}_{\boldsymbol{\lambda}}^{\otimes k} \mathbf{M}(\boldsymbol{\lambda})\right|_{\boldsymbol{\lambda}=\mathbf{0}}=\left.(-i)^{k} \mathbf{D}_{\boldsymbol{\lambda}}^{\otimes k} \mathcal{F}_{\mathbf{X}}(\boldsymbol{\lambda})\right|_{\boldsymbol{\lambda}=\mathbf{0}}
$$


The Cumulant Generating Function (CGF) of $f(\mathbf{X})$ is given by,

$$
\mathbf{C}(\boldsymbol{\lambda})=\ln \mathbf{M}(\boldsymbol{\lambda})=\sum_{k=1}^{\infty} \mathbf{c}(k, d)^{\prime} \frac{\boldsymbol{\lambda}^{\otimes k}}{k !}
$$

where, $\mathbf{c}(k, d)$ is the $k^{t h}$ order cumulant vector of dimension $d^{k} \times 1$.

The Cumulant Generating Function (CGF) of $f(\mathbf{X})$ can also be defined using the characteristic function, as under:

$$
\mathcal{C}(\boldsymbol{\lambda})=\ln \mathcal{F}(\boldsymbol{\lambda})=\sum_{k=1}^{\infty} \mathbf{c}(k, d)^{\prime} \frac{(i \boldsymbol{\lambda})^{\otimes k}}{k !}
$$

Assuming $\mathbf{C}(\boldsymbol{\lambda})$ and $\mathcal{C}(\boldsymbol{\lambda})$ have been expanded using Taylor series,

$$
\mathbf{c}(k, d)=\left.\mathbf{D}_{\boldsymbol{\lambda}}^{\otimes k} \mathbf{C}(\boldsymbol{\lambda})\right|_{\boldsymbol{\lambda}=\mathbf{0}}=\left.(-i)^{k} \mathbf{D}_{\boldsymbol{\lambda}}^{\otimes k} \mathcal{C}_{\mathbf{x}}(\boldsymbol{\lambda})\right|_{\boldsymbol{\lambda}=\mathbf{0}}
$$

Significantly, this section has derived the moments and the cumulants of a random vector in vector notations.

\subsection{Relation between the cumulant vectors and the moment vectors}

The relation between the moments and the cumulants is given by combining Equation (14) and Equation (16) as below:

$$
\mathbf{M}(\boldsymbol{\lambda})=\sum_{k=0}^{\infty} \mathbf{m}(k, d)^{\prime} \frac{\boldsymbol{\lambda}^{\otimes k}}{k !}=\exp \left(\sum_{k=1}^{\infty} \mathbf{c}(k, d)^{\prime} \frac{\boldsymbol{\lambda}^{\otimes k}}{k !}\right)
$$

For $k=1$, using Equation (15), we get:

$$
\mathbf{m}(1, d)=\left.\mathbf{D}_{\boldsymbol{\lambda}}^{\otimes 1} \mathbf{M}(\boldsymbol{\lambda})\right|_{\boldsymbol{\lambda}=\mathbf{0}}
$$

Applying K-derivative $\left(\mathbf{D}_{\lambda}^{\otimes}\right)$ to Equation (19),

$$
\begin{aligned}
\left.\sum_{p=1}^{\infty} \mathbf{m}(p, d)^{\prime} \frac{\left(\boldsymbol{\lambda}^{\otimes(p-1)} \otimes \mathbf{I}_{d}\right)}{(p-1) !}\right|_{\boldsymbol{\lambda}=\mathbf{0}}= & \sum_{p=1}^{\infty} \mathbf{c}(p, d)^{\prime} \frac{\left(\boldsymbol{\lambda}^{\otimes(p-1)} \otimes \mathbf{I}_{d}\right)}{(p-1) !} \\
& \left.\otimes \exp \left(\sum_{q=1}^{\infty} \mathbf{c}(q, d)^{\prime} \frac{\boldsymbol{\lambda}^{\otimes q}}{q !}\right)\right|_{\boldsymbol{\lambda}=\mathbf{0}} \\
\Rightarrow \mathbf{m}(1, d) & =\mathbf{c}(1, d)
\end{aligned}
$$

Similarly, based on Equation (15), for any $\mathrm{k}$ taking $k^{\text {th }}$ order K-derivative of above Equation (19) on both sides relates $\mathbf{m}(k, d)$ with $\mathbf{c}(k, d)$. For example, the cases for $k=2$ and $k=3$ are shown in Appendix D.2. Overall, the vector 
moments in terms of the vector cumulants can be summarized as under:

$$
\begin{aligned}
\mathbf{m}(1, d)= & \mathbf{c}(1, d) \\
\mathbf{m}(2, d)= & \mathbf{c}(2, d)+\mathbf{c}(1, d)^{\otimes 2} \\
\mathbf{m}(3, d)= & \mathbf{c}(3, d)+3 \mathbf{c}(2, d) \otimes \mathbf{c}(1, d)+\mathbf{c}(1, d)^{\otimes 3} \\
\mathbf{m}(4, d)= & \mathbf{c}(4, d)+4 \mathbf{c}(3, d) \otimes \mathbf{c}(1, d)+3 \mathbf{c}(2, d)^{\otimes 2}+6 \mathbf{c}(2, d) \\
& \otimes \mathbf{c}(1, d)^{\otimes 2}+\mathbf{c}(1, d)^{\otimes 4} \\
\mathbf{m}(5, d)= & \mathbf{c}(5, d)+5 \mathbf{c}(4, d) \otimes \mathbf{c}(1, d)+10 \mathbf{c}(3, d) \otimes \mathbf{c}(2, d) \\
& +10 \mathbf{c}(3, d) \otimes \mathbf{c}(1, d)^{\otimes 2}+15 \mathbf{c}(2, d)^{\otimes 2} \otimes \mathbf{c}(1, d) \\
+ & 10 \mathbf{c}(2, d) \otimes \mathbf{c}(1, d)^{\otimes 3}+\mathbf{c}(1, d)^{\otimes 5} \\
\mathbf{m}(6, d)= & \mathbf{c}(6, d)+6 \mathbf{c}(5, d) \otimes \mathbf{c}(1, d)+15 \mathbf{c}(4, d) \otimes \mathbf{c}(2, d) \\
& +15 \mathbf{c}(4, d) \otimes \mathbf{c}(1, d)^{\otimes 2}+10 \mathbf{c}(3, d)^{\otimes 2}+60 \mathbf{c}(3, d) \otimes \mathbf{c}(2, d) \\
& \otimes \mathbf{c}(1, d)+20 \mathbf{c}(3, d) \otimes \mathbf{c}(1, d)^{\otimes 2}+15 \mathbf{c}(2, d)^{\otimes 3} \\
+ & 45 \mathbf{c}(2, d)^{\otimes 2} \otimes \mathbf{c}(1, d)^{\otimes 2}+15 \mathbf{c}(2, d) \otimes \mathbf{c}(1, d)^{\otimes 4}+\mathbf{c}(1, d)^{\otimes 6}
\end{aligned}
$$

The above set of equations can be represented through more compact formulas as under. The Equation (21) gives generalized $k^{t h}$ order d-variate cumulant vector in terms of the moment vectors and the Equation (22) gives the vice-a-versa.

$$
\begin{array}{r}
\mathbf{m}(k, d)=\sum_{p=0}^{k-1}\left(\begin{array}{c}
k-1 \\
p
\end{array}\right) \mathbf{K}_{\mathfrak{p} h \leftrightarrow l}^{-1} \mathbf{c}(k-p, d) \otimes \mathbf{m}(p, d) \\
\mathbf{c}(k, d)=\mathbf{m}(k, d)-\sum_{p=1}^{k-1}\left(\begin{array}{c}
k-1 \\
p
\end{array}\right) \mathbf{K}_{\mathfrak{p} h \leftrightarrow l}^{-1} \mathbf{c}(k-p, d) \otimes \mathbf{m}(p, d)
\end{array}
$$

where, $\mathbf{K}_{\mathfrak{p} h \leftrightarrow l}^{-1}$ is a specific commutation matrix with corresponding dimensions that changes the place of the cumulants for Kronecker product such that the expression has decreasing order cumulants from left to the right, i.e. the higher order cumulant vector on the left and the lower order cumulant vector on the right. As the Kronecker products are non-commutative, without using the commutation matrices it would have been impossible to derive the compact formula. The derived multivariate expressions reduce to the following expressions for dimension $d=1$ and are exactly same as those derived in (Berberan-Santos, 2007).

$$
\begin{aligned}
& \qquad(k, 1)=m(k, 1)-\sum_{p=1}^{k-1}\left(\begin{array}{c}
k-1 \\
p
\end{array}\right) c(k-p, 1) m(p, 1) \\
& \text { or more simply, } c_{k}=m_{k}-\sum_{p=1}^{k-1}\left(\begin{array}{c}
k-1 \\
p
\end{array}\right) c_{k-p} m_{p}
\end{aligned}
$$

Thus, the derived multivariate expressions in Equation (20) are elementary vector extensions to those for univariate. 


\section{Multivariate $p d f$ representation in terms of the cumulants}

From Equation (11) and Equation (17), the multivariate $p d f f(\mathbf{x})$ can be written as:

$$
f(\mathbf{x})=\mathrm{F}^{-1}\left(\mathrm{e}^{\mathcal{C}(\boldsymbol{\lambda})}\right)=\left(\frac{1}{2 \pi}\right)^{d} \int_{\mathbb{R}^{d}} \exp \left(\sum_{k=1}^{\infty} \mathbf{c}(k, d)^{\prime} \frac{(i \boldsymbol{\lambda})^{\otimes k}}{k !}\right) \exp \left(-i \mathbf{x}^{\prime} \boldsymbol{\lambda}\right) d \boldsymbol{\lambda}
$$

As $p d f$ is a real function and $\operatorname{Re}\left(e^{A+i B} e^{-i C}\right)=e^{A} \cos (B-C)=e^{A} \cos (C-B)$, the Equation (24) can be re-written as:

$$
\begin{aligned}
f(\mathbf{x})= & \frac{1}{(2 \pi)^{d}} \int_{\mathbb{R}^{d}} \exp \left(\sum_{k=1}^{\infty} \frac{\mathbf{c}(2 k, d)^{\prime}}{2 k !}(i \boldsymbol{\lambda})^{\otimes 2 k}\right) \\
& \cos \left(\mathbf{x}^{\prime} \boldsymbol{\lambda}+\sum_{k=1}^{\infty} \frac{\mathbf{c}(2 k-1, d)^{\prime}}{(2 k-1) !}(i)^{2 k}(\boldsymbol{\lambda})^{\otimes(2 k-1)}\right) d \boldsymbol{\lambda}
\end{aligned}
$$

The integrand in this equation is an even function. So,

$$
\begin{aligned}
f(\mathbf{x})= & \frac{1}{(\pi)^{d}} \int_{\left(\mathbb{R}^{+}\right)^{d}} \exp \left(\sum_{k=1}^{\infty} \frac{\mathbf{c}(2 k, d)^{\prime}}{2 k !}(i \boldsymbol{\lambda})^{\otimes 2 k}\right) \\
& \cos \left(\mathbf{x}^{\prime} \boldsymbol{\lambda}+\sum_{k=1}^{\infty} \frac{\mathbf{c}(2 k-1, d)^{\prime}}{(2 k-1) !}(i)^{2 k}(\boldsymbol{\lambda})^{\otimes(2 k-1)}\right) d \boldsymbol{\lambda}
\end{aligned}
$$

where, $\mathbb{R}^{+}=\{x \in \mathbb{R}: x \geq 0\}$. The Equation (25) and the Equation (26) give a multivariate $p d f$ in terms of the cumulants. As they are derived using Taylor series expansion, the infinite order differentiability is an implicit assumption.

The equations can be verified using known $p d f$ examples with finite number of moments and cumulants. Let say, the impulse delta density function has only the first order cumulant being non-zero and all other higher order cumulants are zero. Using this knowledge in Equation (25),

$$
\begin{aligned}
f(\mathbf{x}) & =\frac{1}{(2 \pi)^{d}} \int_{\mathbb{R}^{d}} \cos \left((\mathbf{x}-\mathbf{c}(1, d))^{\prime} \boldsymbol{\lambda}\right) d \boldsymbol{\lambda} \\
& =\delta(\mathbf{x}-\mathbf{c}(1, d)) \quad(\text { shifted impulse delta function) }
\end{aligned}
$$

Let's take another example, the Gaussian density function has first two order cumulants nonzero and all other order cumulants are zero. Using this knowledge in Equation (25),

$$
\begin{aligned}
f(\mathbf{x}) & =\frac{1}{(2 \pi)^{d}} \int_{\mathbb{R}^{d}} \exp \left(-\frac{\mathbf{c}(2, d)^{\prime}}{2} \boldsymbol{\lambda}^{\otimes 2}\right) \cos \left((\mathbf{x}-\mathbf{c}(1, d))^{\prime} \boldsymbol{\lambda}\right) d \boldsymbol{\lambda} \\
& =G(\mathbf{x}) \quad(\text { See Appendix D.3 for proof })
\end{aligned}
$$




\section{The multivariate Hermite polynomials in integral form}

An interesting application of the integral form of multivariate $p d f$ representation is achieved in this section. The multivariate Gaussian expressed as in Equation (28) is used to derive it's differentials and Hermite polynomials in a simple way. Taking $k^{\text {th }}$ K-derivative of $G(\mathbf{x})$,

$$
\begin{aligned}
G^{(k)}(\mathbf{x}):= & \mathbf{D}_{\mathbf{x}}^{\otimes k} G(\mathbf{x})=\frac{1}{(2 \pi)^{d}} \int_{\mathbb{R}^{d}} \boldsymbol{\lambda}^{\otimes k} \exp \left(-\frac{\mathbf{c}(2, d)^{\prime}}{2} \boldsymbol{\lambda}^{\otimes 2}\right) \\
& \cos \left((\mathbf{x}-\mathbf{c}(1, d))^{\prime} \boldsymbol{\lambda}+\frac{k \pi}{2}\right) d \boldsymbol{\lambda}
\end{aligned}
$$

The multivariate Hermite polynomials defined by Holmquist (1996) are defined as under:

$$
\begin{aligned}
\mathbf{H}_{k}\left(\mathbf{x} ; \mathbf{0}, \mathbf{C}_{\mathbf{x}}\right) & =\left[G\left(\mathbf{x} ; \mathbf{0}, \mathbf{C}_{\mathbf{x}}\right)\right]^{-1}(-1)^{k}\left(\mathbf{C}_{\mathbf{x}} \mathbf{D}_{x}\right)^{\otimes k} G\left(\mathbf{x} ; \mathbf{0}, \mathbf{C}_{\mathbf{x}}\right) \\
\text { where, } G\left(\mathbf{x} ; \mathbf{0}, \mathbf{C}_{\mathbf{x}}\right) & =\left|\mathbf{C}_{\mathbf{x}}\right|^{-1 / 2}(2 \pi)^{-d / 2} \exp \left(-\frac{1}{2} \mathbf{x}^{\prime} \mathbf{C}_{\mathbf{x}}^{-1} \mathbf{x}\right) \\
& =\left|\mathbf{C}_{\mathbf{x}}\right|^{-1 / 2}(2 \pi)^{-d / 2} \exp \left(-\frac{1}{2}\left(V e c \mathbf{C}_{\mathbf{x}}^{-1}\right)^{\prime} \mathbf{x}^{\otimes 2}\right)
\end{aligned}
$$

This is equivalent to the 1-dimensional definition of Hermite polynomials ${ }^{3}$ by Rodrigues's formula in Equation (32), except the introduction of matrix $\mathbf{C}_{\mathbf{x}}$.

$$
H_{k}(x)=[G(x)]^{-1}(-1)^{k} \frac{d^{k}}{d x^{k}} G(x) \text { where, } G(x)=\frac{1}{2 \pi} \mathrm{e}^{-\frac{1}{2} x^{2}}
$$

Using Equation (29), the Equation (30) for multivariate Hermite polynomials is rewritten as:

$$
\begin{gathered}
\mathbf{H}_{k}\left(\mathbf{x} ; \mathbf{0}, \mathbf{C}_{\mathbf{x}}\right)=(2 \pi)^{d / 2}\left|\mathbf{C}_{\mathbf{x}}\right|^{1 / 2}(-1)^{k}\left(\mathbf{C}_{\mathbf{x}}\right)^{\otimes k} \exp \left(\frac{1}{2}\left(V e c \mathbf{C}_{\mathbf{x}}^{-1}\right)^{\prime} \mathbf{x}^{\otimes 2}\right) \frac{1}{(2 \pi)^{d}} \\
\int_{\mathbb{R}^{d}} \boldsymbol{\lambda}^{\otimes k} \exp \left(-\frac{\mathbf{c}(2, d)^{\prime}}{2} \boldsymbol{\lambda}^{\otimes 2}\right) \cos \left((\mathbf{x}-\mathbf{c}(1, d))^{\prime} \boldsymbol{\lambda}+\frac{k \pi}{2}\right) d \boldsymbol{\lambda}
\end{gathered}
$$

Taking $\mathbf{C}_{\mathbf{x}}=\mathbf{I}_{d}$, where $\mathbf{I}_{d}$ is $d \times d$ identity matrix; using the property $\left(V e c \mathbf{I}_{d}\right)^{\prime} \mathbf{x}^{\otimes 2}=$ $\mathbf{x}^{\prime} \mathbf{x}$ and using change of variable as $\boldsymbol{\lambda} / \sqrt{2}=\mathbf{u}$ - the integral form of multivariate Hermite polynomials is obtained as under:

$$
\begin{aligned}
\mathbf{H}_{k}\left(\mathbf{x} ; \mathbf{0}, \mathbf{I}_{d}\right)= & (2)^{\frac{k+d+1}{2}}(\pi)^{-d / 2} \exp \left(\frac{1}{2} \mathbf{x}^{\prime} \mathbf{x}\right) \\
& \int_{\mathbb{R}^{d}} \mathbf{u}^{\otimes k} \exp \left(-\mathbf{u}^{\prime} \mathbf{u}\right) \cos \left(\sqrt{2} \mathbf{x}^{\prime} \mathbf{u}-\frac{k \pi}{2}\right) d \mathbf{u}
\end{aligned}
$$

\footnotetext{
${ }^{3}$ This is the 'probabilists' Hermite polynomials and not the 'physicists' Hermite polynomials used by Berberan-Santos (2007).
} 
The result in Equation (29) can also be obtained using Equation (28) and applying the derivative property of Fourier transform (F). The $k^{\text {th }}$ derivative of $G(x)$ is given by,

$$
\begin{aligned}
G^{(k)}(\mathbf{x}) & =\mathrm{F}^{-1}\left(\mathrm{~F}\left(G^{(k)}(\mathbf{x})\right)\right) \\
& =\frac{1}{(2 \pi)^{d}} \int_{\mathbb{R}^{d}}(i \boldsymbol{\lambda})^{\otimes k} \mathrm{~F}(G(\mathbf{x})) \exp ^{-i \mathbf{x}^{\prime} \boldsymbol{\lambda}} d \boldsymbol{\lambda} \\
& =\frac{1}{(2 \pi)^{d}} \int_{\mathbb{R}^{d}} \boldsymbol{\lambda}^{\otimes k} \exp \left(-\frac{\mathbf{c}(2, d)^{\prime}}{2} \boldsymbol{\lambda}^{\otimes 2}\right) \cos \left((\mathbf{x}-\mathbf{c}(1, d))^{\prime} \boldsymbol{\lambda}+\frac{k \pi}{2}\right) d \boldsymbol{\lambda}
\end{aligned}
$$

\section{Multivariate Gram-Charlier A series}

Till now, the article has derived - an unknown $p d f$ expressed in terms of its cumulants in Equation (25); the Gaussian density function expressed in terms of its cumulants in Equation (28) and the Hermite polynomials in Equation (34). Based on them, the multivariate Gram Charlier A series that expresses an unknown $p d f$ using Gaussian density as a reference can be obtained. The expansion assumes first and second order cumulants being same for both the unknown $p d f$ and the reference $p d f$. Using the expansion $\exp (A+B) \cos (C+D)=$ $\exp (A) \exp (B)(\cos C \cos D-\sin C \sin D)$, the Equation (25) can be re-written as:

$$
\begin{aligned}
f(\mathbf{x})= & \frac{1}{(2 \pi)^{d}} \int_{\mathbb{R}^{d}} \exp \left(-\frac{\mathbf{c}(2, d)^{\prime}}{2} \boldsymbol{\lambda}^{\otimes 2}\right) \exp \left(\sum_{k=2}^{\infty} \frac{\mathbf{c}(2 k, d)^{\prime}}{2 k !}(i \boldsymbol{\lambda})^{\otimes 2 k}\right) \\
& \left\{\cos \left((\mathbf{x}-\mathbf{c}(1, d))^{\prime} \boldsymbol{\lambda}\right) \cos \left(\sum_{k=2}^{\infty} \frac{\mathbf{c}(2 k-1, d)^{\prime}}{(2 k-1) !}(i)^{2 k}(\boldsymbol{\lambda})^{\otimes(2 k-1)}\right)\right. \\
& \left.-\sin \left((\mathbf{x}-\mathbf{c}(1, d))^{\prime} \boldsymbol{\lambda}\right) \sin \left(\sum_{k=2}^{\infty} \frac{\mathbf{c}(2 k-1, d)^{\prime}}{(2 k-1) !}(i)^{2 k}(\boldsymbol{\lambda})^{\otimes(2 k-1)}\right)\right\} d \boldsymbol{\lambda}
\end{aligned}
$$

Using the expansions in Appendix C, parts of the Equation (38) can be approximated upto maximum $6^{\text {th }}$-order statistics as under:

$$
\begin{aligned}
& \exp \left(\sum_{k=2}^{\infty} \frac{\mathbf{c}(2 k, d)^{\prime}}{2 k !}(i \boldsymbol{\lambda})^{\otimes 2 k}\right) \cos \left(\sum_{k=2}^{\infty} \frac{\mathbf{c}(2 k-1, d)^{\prime}}{(2 k-1) !}(i)^{2 k}(\boldsymbol{\lambda})^{\otimes(2 k-1)}\right) \\
& =1+\left(\frac{\mathbf{c}(4, d)^{\prime} \boldsymbol{\lambda}^{\otimes 4}}{4 !}-\frac{\mathbf{c}(6, d)^{\prime} \boldsymbol{\lambda}^{\otimes 6}}{6 !}\right)-\frac{1}{2}\left(\frac{\mathbf{c}(3, d)^{\prime} \boldsymbol{\lambda}^{\otimes 3}}{3 !}\right)^{\otimes 2}+\ldots \\
& \exp \left(\sum_{k=2}^{\infty} \frac{\mathbf{c}(2 k, d)^{\prime}}{2 k !}(i \boldsymbol{\lambda})^{\otimes 2 k}\right) \sin \left(\sum_{k=2}^{\infty} \frac{\mathbf{c}(2 k-1, d)^{\prime}}{(2 k-1) !}(-i)^{2 k}(\boldsymbol{\lambda})^{\otimes(2 k-1)}\right) \\
& \quad=\frac{\mathbf{c}(3, d)^{\prime} \boldsymbol{\lambda}^{\otimes 3}}{3 !}-\frac{\mathbf{c}(5, d)^{\prime} \boldsymbol{\lambda}^{\otimes 5}}{5 !}+\ldots
\end{aligned}
$$


Using above Equation (39) and Equation (40), the Equation (38) can be rewritten as:

$$
\begin{aligned}
f(\mathbf{x})= & \frac{1}{(2 \pi)^{d}} \int_{\mathbb{R}^{d}}\left\{\exp \left(-\frac{\mathbf{c}(2, d)^{\prime}}{2} \boldsymbol{\lambda}^{\otimes 2}\right) \cos \left((\mathbf{x}-\mathbf{c}(1, d))^{\prime} \boldsymbol{\lambda}\right)\right. \\
& \left.\left(1+\frac{\mathbf{c}(4, d)^{\prime} \boldsymbol{\lambda}^{\otimes 4}}{4 !}-\frac{1}{6 !}\left(\mathbf{c}(6, d)-10 \mathbf{c}(3, d)^{\otimes 2}\right)^{\prime} \boldsymbol{\lambda}^{\otimes 6}+\ldots\right)\right\} d \boldsymbol{\lambda} \\
& -\frac{1}{(2 \pi)^{d}} \int_{\mathbb{R}^{d}}\left\{\exp \left(-\frac{\mathbf{c}(2, d)^{\prime}}{2} \boldsymbol{\lambda}^{\otimes 2}\right) \cos \left((\mathbf{x}-\mathbf{c}(1, d))^{\prime} \boldsymbol{\lambda}-\frac{\pi}{2}\right)\right. \\
& \left.\left(\frac{\mathbf{c}(3, d)^{\prime} \boldsymbol{\lambda}^{\otimes 3}}{3 !}-\frac{\mathbf{c}(5, d)^{\prime} \boldsymbol{\lambda}^{\otimes 5}}{5 !}+\ldots\right)\right\} d \boldsymbol{\lambda}
\end{aligned}
$$

Using the Equation (29) for derivatives of Gaussian defined, the above Equation can be simplified as:

$$
\begin{aligned}
f(\mathbf{x})= & G(\mathbf{x})-\frac{\mathbf{c}(3, d)^{\prime}}{3 !} G^{(3)}(\mathbf{x})+\frac{\mathbf{c}(4, d)^{\prime}}{4 !} G^{(4)}(\mathbf{x})-\frac{\mathbf{c}(5, d)^{\prime}}{5 !} G^{(5)}(\mathbf{x}) \\
& +\frac{\mathbf{c}(6, d)^{\prime}+10 \mathbf{c}(3, d)^{\otimes 2^{\prime}}}{6 !} G^{(6)}(\mathbf{x})+\ldots
\end{aligned}
$$

The Equation (42) is the Gram-Charlier A series expressed directly in terms of the cumulants and the derivatives of the Gaussian pdf. Usually, the GCA is represented in terms of the Hermite polynomials. So, the GCA expansion (Equation (42)) in terms of the Hermite polynomials; either using definition in Equation (30) or using $\mathbf{H}_{k}\left(\mathbf{x} ; \mathbf{0}, \mathbf{C}_{\mathbf{x}}^{-1}\right)$ derived in Equation (33); can be re-written as:

$$
\begin{aligned}
f(\mathbf{x})= & G(\mathbf{x})\left[1+\frac{\mathbf{c}(3, d)^{\prime}}{3 !}\left(\mathbf{C}_{\mathbf{x}}^{-1}\right)^{\otimes 3} \mathbf{H}_{3}\left(\mathbf{x} ; \mathbf{0}, \mathbf{C}_{\mathbf{x}}\right)+\frac{\mathbf{c}(4, d)^{\prime}}{4 !}\left(\mathbf{C}_{\mathbf{x}}^{-1}\right)^{\otimes 4} \mathbf{H}_{4}\left(\mathbf{x} ; \mathbf{0}, \mathbf{C}_{\mathbf{x}}\right)\right. \\
& +\frac{\mathbf{c}(5, d)^{\prime}}{5 !}\left(\mathbf{C}_{\mathbf{x}}^{-1}\right)^{\otimes 5} \mathbf{H}_{5}\left(\mathbf{x} ; \mathbf{0}, \mathbf{C}_{\mathbf{x}}\right)+\frac{\mathbf{c}(6, d)^{\prime}+10 \mathbf{c}(3, d)^{\otimes 2^{\prime}}}{6 !}\left(\mathbf{C}_{\mathbf{x}}^{-1}\right)^{\otimes 6} \\
& \left.\mathbf{H}_{6}\left(\mathbf{x} ; \mathbf{0}, \mathbf{C}_{\mathbf{x}}\right)+\ldots\right] \\
f(\mathbf{x})= & G(\mathbf{x})\left[1+\frac{\mathbf{c}(3, d)^{\prime}}{3 !} \mathbf{H}_{3}\left(\mathbf{x} ; \mathbf{0}, \mathbf{I}_{d}\right)+\frac{\mathbf{c}(4, d)^{\prime}}{4 !} \mathbf{H}_{4}\left(\mathbf{x} ; \mathbf{0}, \mathbf{I}_{d}\right)+\frac{\mathbf{c}(5, d)^{\prime}}{5 !} \mathbf{H}_{5}\left(\mathbf{x} ; \mathbf{0}, \mathbf{I}_{d}\right)\right. \\
& \left.+\frac{\mathbf{c}(6, d)^{\prime}+10 \mathbf{c}(3, d)^{\otimes 2^{\prime}}}{6 !} \mathbf{H}_{6}\left(\mathbf{x} ; \mathbf{0}, \mathbf{I}_{d}\right)+\ldots\right]
\end{aligned}
$$

Finally, the GCA series, in vector notations, can be expressed either using $k^{t h}$ order derivative of Gaussian $\left(G_{k}(\mathbf{x})\right)$ or using $k^{\text {th }}$ order vector Hermite polynomials $\left(\mathbf{H}_{k}(\mathbf{x})\right)$ as under:

$$
\begin{aligned}
f(\mathbf{x}) & =\sum_{k=0}^{\infty}(-1)^{k} \frac{\mathbf{c}(k, d)^{\prime}}{k !} G^{(k)}(\mathbf{x}) \\
& =\sum_{k=0}^{\infty} \frac{\mathbf{c}(k, d)^{\prime}}{k !} \mathbf{H}_{k}\left(\mathbf{x} ; \mathbf{0}, \mathbf{I}_{d}\right)
\end{aligned}
$$




\section{Multivariate Generalized Gram-Charlier series}

To derive the generalized Gram-Charlier series, an unknown $p d f f(\mathbf{x})$ need be represented in terms of any known reference $p d f \psi(\mathbf{x})$, where both the $p d f \mathrm{~s}$ are represented in terms of their cumulants. Let the $k^{t h}$ order cumulant vector of the reference $p d f \psi(\mathbf{x})$ be $\mathbf{c}_{r}(k, d)$. Then, the $k^{t h}$ order cumulant difference vector $\boldsymbol{\delta}(k, d)$ is: $\boldsymbol{\delta}(k, d)=\mathbf{c}(k, d)-\mathbf{c}_{r}(k, d), \forall k$. Using $\boldsymbol{\delta}(k, d)$, the Equation (25) can be re-written as under:

$$
\begin{aligned}
f(\mathbf{x})= & \frac{1}{(2 \pi)^{d}} \int_{\mathbb{R}^{d}} \exp \left(\sum_{k=1}^{\infty} \frac{\mathbf{c}_{r}(2 k, d)^{\prime}}{2 k !}(i \boldsymbol{\lambda})^{\otimes 2 k}\right) \exp \left(\sum_{k=1}^{\infty} \frac{\boldsymbol{\delta}(2 k, d)^{\prime}}{2 k !}(i \boldsymbol{\lambda})^{\otimes 2 k}\right) \\
& \cos \left(\mathbf{x}^{\prime} \boldsymbol{\lambda}+\left(\sum_{k=1}^{\infty} \frac{\mathbf{c}_{r}(2 k-1, d)}{(2 k-1) !}+\sum_{k=1}^{\infty} \frac{\boldsymbol{\delta}(2 k-1, d)}{(2 k-1) !}\right)^{\prime}(i)^{2 k}(\boldsymbol{\lambda})^{\otimes(2 k-1)}\right) d \boldsymbol{\lambda} \\
= & \frac{1}{(2 \pi)^{d}} \int_{\mathbb{R}^{d}} \exp \left(\sum_{k=1}^{\infty} \frac{\mathbf{c}_{r}(2 k, d)^{\prime}}{2 k !}(i \boldsymbol{\lambda})^{\otimes 2 k}\right) \exp \left(\sum_{k=1}^{\infty} \frac{\boldsymbol{\delta}(2 k, d)^{\prime}}{2 k !}(i \boldsymbol{\lambda})^{\otimes 2 k}\right) \\
& \left\{\operatorname { c o s } ( \mathbf { x } ^ { \prime } \boldsymbol { \lambda } + \sum _ { k = 1 } ^ { \infty } \frac { \mathbf { c } _ { r } ( 2 k - 1 , d ) ^ { \prime } } { ( 2 k - 1 ) ^ { \prime } } ( i ) ^ { 2 k } ( \boldsymbol { \lambda } ) ^ { \otimes ( 2 k - 1 ) } ) \operatorname { c o s } \left(\sum_{k=1}^{\infty} \frac{\boldsymbol{\delta}(2 k-1, d)^{\prime}}{(2 k-1) !}\right.\right. \\
& \left.(i)^{2 k}(\boldsymbol{\lambda})^{\otimes(2 k-1)}\right)-\sin \left(\mathbf{x}^{\prime} \boldsymbol{\lambda}+\sum_{k=1}^{\infty} \frac{\mathbf{c}_{r}(2 k-1, d)^{\prime}}{(2 k-1) !}(i)^{2 k}(\boldsymbol{\lambda})^{\otimes(2 k-1)}\right) \\
& \left.\sin \left(\sum_{k=1}^{\infty} \frac{\boldsymbol{\delta}(2 k-1, d)^{\prime}}{(2 k-1) !}(i)^{2 k}(\boldsymbol{\lambda})^{\otimes(2 k-1)}\right)\right\} d \boldsymbol{\lambda}
\end{aligned}
$$


Using the expansions in Appendix C, parts of the Equation (48) can be approximated upto maximum $6^{\text {th }}$-order statistics as under:

$$
\begin{aligned}
& \exp \left(\sum_{k=1}^{\infty} \frac{\boldsymbol{\delta}(2 k, d)^{\prime}}{2 k !}(i \boldsymbol{\lambda})^{\otimes 2 k}\right) \cos \left(\sum_{k=1}^{\infty} \frac{\boldsymbol{\delta}(2 k-1, d)^{\prime}}{(2 k-1) !}(i)^{2 k}(\boldsymbol{\lambda})^{\otimes(2 k-1)}\right) \\
&=1+\left(\sum_{k=1}^{\infty} \frac{\boldsymbol{\delta}(2 k, d)^{\prime}}{2 k !}(i \boldsymbol{\lambda})^{\otimes 2 k}\right)+\frac{1}{2}\left(\sum_{k=1}^{\infty} \frac{\boldsymbol{\delta}(2 k, d)^{\prime}}{2 k !}(i \boldsymbol{\lambda})^{\otimes 2 k}\right)^{\otimes 2} \\
&-\frac{1}{2}\left(\sum_{k=1}^{\infty} \frac{\boldsymbol{\delta}(2 k-1, d)^{\prime}}{(2 k-1) !}(i)^{2 k}(\boldsymbol{\lambda})^{\otimes(2 k-1)}\right)^{\otimes 2}-\ldots \\
&=+\left(-\frac{\boldsymbol{\delta}(2, d)^{\prime}}{2 !} \boldsymbol{\lambda}^{\otimes 2}+\frac{\boldsymbol{\delta}(4, d)^{\prime}}{4 !} \boldsymbol{\lambda}^{\otimes 4}-\frac{\boldsymbol{\delta}(6, d)^{\prime}}{6 !} \boldsymbol{\lambda}^{\otimes 6}\right)+\frac{1}{2}\left(-\frac{\boldsymbol{\delta}(2, d)^{\prime}}{2 !} \boldsymbol{\lambda}^{\otimes 2}\right)^{\otimes 2} \\
&-\frac{1}{2}\left(-\boldsymbol{\delta}(1, d)^{\prime} \boldsymbol{\lambda}+\frac{\boldsymbol{\delta}(3, d)^{\prime}}{3 !} \boldsymbol{\lambda}^{\otimes 3}\right)^{\otimes 2}+\frac{1}{6}\left(-\frac{\boldsymbol{\delta}(2, d)^{\prime}}{2 !} \boldsymbol{\lambda}^{\otimes 2}\right)^{\otimes 3}-\frac{1}{2}\left(\left(-\frac{\boldsymbol{\delta}(2, d)^{\prime}}{2 !} \boldsymbol{\lambda}^{\otimes 2}\right.\right. \\
&\left.\left.+\frac{\boldsymbol{\delta}(4, d)^{\prime}}{4 !} \boldsymbol{\lambda}^{\otimes 4}\right) \otimes\left(\boldsymbol{\delta}(1, d)^{\prime} \boldsymbol{\lambda}\right)^{\otimes 2}\right)-\frac{1}{4}\left(\left(-\frac{\boldsymbol{\delta}(2, d)^{\prime}}{2 !} \boldsymbol{\lambda}^{\otimes 2}\right)^{\otimes 2} \otimes\left(\boldsymbol{\delta}(1, d)^{\prime} \boldsymbol{\lambda}\right)^{\otimes 2}\right) \\
&+\frac{1}{4 !}\left(-\frac{\boldsymbol{\delta}(2, d)^{\prime}}{2 !} \boldsymbol{\lambda}^{\otimes 2}\right)^{\otimes 4}-\frac{1}{4 !}\left(-\boldsymbol{\delta}(1, d)^{\prime} \boldsymbol{\lambda}\right)^{\otimes 4}-\left(\frac{\boldsymbol{\delta}(2, d)^{\prime}}{2 !} \boldsymbol{\lambda}^{\otimes 2} \otimes \boldsymbol{\delta}(1, d)^{\prime} \boldsymbol{\lambda}^{\otimes 4}\right)+\ldots \\
&= 1-\frac{1}{2}\left(\boldsymbol{\delta}(1, d)^{\otimes 2}+\boldsymbol{\delta}(2, d)\right)^{\prime} \boldsymbol{\lambda}^{\otimes 2}+\frac{1}{4 !}\left(\boldsymbol{\delta}(1, d)^{\otimes 4}+6 \boldsymbol{\delta}(2, d) \otimes \boldsymbol{\delta}(1, d)^{\otimes 2}\right. \\
&\left.+3 \boldsymbol{\delta}(2, d)^{\otimes 2}+4 \boldsymbol{\delta}(3, d) \otimes \boldsymbol{\delta}(1, d)+\boldsymbol{\delta}(4, d)\right)^{\prime} \boldsymbol{\lambda}^{\otimes 4}-\frac{1}{6 !}\left(\boldsymbol{\delta}(1, d)^{\otimes 6}+15 \boldsymbol{\delta}(2, d)^{\otimes 3}\right. \\
&+10 \boldsymbol{\delta}(3, d)^{\otimes 2}+15 \boldsymbol{\delta}(4, d) \otimes \boldsymbol{\delta}(2, d)+15 \boldsymbol{\delta}(4, d) \otimes \boldsymbol{\delta}(1, d)^{\otimes 2}+20 \boldsymbol{\delta}(3, d) \otimes \boldsymbol{\delta}(1, d)^{\otimes 2} \\
&+15 \boldsymbol{\delta}(2, d) \otimes \boldsymbol{\delta}(1, d)^{\otimes 4}+45 \boldsymbol{\delta}(2, d)^{\otimes 2} \otimes \boldsymbol{\delta}(1, d)^{\otimes 2}+6 \boldsymbol{\delta}(5, d) \otimes \boldsymbol{\delta}(1, d) \\
&+60 \boldsymbol{\delta}(3, d) \otimes \boldsymbol{\delta}(2, d) \otimes \boldsymbol{\delta}(1, d)+\boldsymbol{\delta}(6, d))^{\prime} \boldsymbol{\lambda}^{\otimes 6}+\ldots \\
&(49)
\end{aligned}
$$


Similarly,

$$
\begin{aligned}
& \exp \left(\sum_{k=1}^{\infty} \frac{\boldsymbol{\delta}(2 k, d)^{\prime}}{2 k !}(i \boldsymbol{\lambda})^{\otimes 2 k}\right) \sin \left(\sum_{k=1}^{\infty} \frac{\boldsymbol{\delta}(2 k-1, d)^{\prime}}{(2 k-1) !}(-i)^{2 k}(\boldsymbol{\lambda})^{\otimes(2 k-1)}\right) \\
&=\left(-\boldsymbol{\delta}(1, d)^{\prime} \boldsymbol{\lambda}+\frac{\boldsymbol{\delta}(3, d)^{\prime}}{3 !} \boldsymbol{\lambda}^{\otimes 3}-\frac{\boldsymbol{\delta}(5, d)^{\prime}}{5 !} \boldsymbol{\lambda}^{\otimes 5}\right)+\left(\frac{\boldsymbol{\delta}(2, d)^{\prime} \boldsymbol{\lambda}^{\otimes 2}}{2 !} \otimes \frac{\boldsymbol{\delta}(1, d)^{\prime} \boldsymbol{\lambda}}{1}\right. \\
&\left.\quad+\frac{\boldsymbol{\delta}(3, d)^{\prime} \boldsymbol{\lambda}^{\otimes 3}}{3 !} \otimes \frac{\boldsymbol{\delta}(2, d)^{\prime} \boldsymbol{\lambda}^{\otimes 2}}{2 !}+\frac{\boldsymbol{\delta}(4, d)^{\prime} \boldsymbol{\lambda}^{\otimes 4}}{4 !} \otimes \frac{\boldsymbol{\delta}(1, d)^{\prime} \boldsymbol{\lambda}^{\otimes 1}}{1 !}\right) \\
& \\
& \quad-\frac{1}{2}\left(\frac{\boldsymbol{\delta}(2, d)^{\prime} \boldsymbol{\lambda}^{\otimes 2}}{2 !}\right)^{\otimes 2} \otimes \frac{\boldsymbol{\delta}(1, d)^{\prime} \boldsymbol{\lambda}^{\otimes 1}}{1 !}-\left(-\boldsymbol{\delta}(1, d)^{\prime} \boldsymbol{\lambda}\right)^{\otimes 3}+\ldots \\
&= \boldsymbol{\delta}(1, d)^{\prime} \boldsymbol{\lambda}+\frac{1}{3 !}\left(\boldsymbol{\delta}(3, d)+3 \boldsymbol{\delta}(2, d) \otimes \boldsymbol{\delta}(1, d)+\boldsymbol{\delta}(1, d)^{\otimes 3}\right)^{\prime} \boldsymbol{\lambda}^{\otimes 3}-\frac{1}{5 !}(\boldsymbol{\delta}(5, d) \\
&+5 \boldsymbol{\delta}(4, d) \otimes \boldsymbol{\delta}(1, d)+15 \boldsymbol{\delta}(2, d)^{\otimes 2} \otimes \boldsymbol{\delta}(1, d)+10 \boldsymbol{\delta}(3, d) \otimes \boldsymbol{\delta}(2, d) \\
&\left.+10 \boldsymbol{\delta}(2, d) \otimes \boldsymbol{\delta}(1, d)^{\otimes 3}+10 \boldsymbol{\delta}(3, d) \otimes \boldsymbol{\delta}(1, d)^{\otimes 2}+\boldsymbol{\delta}(1, d)^{\otimes 5}\right)^{\prime} \boldsymbol{\lambda}^{\otimes 5} \ldots(50)
\end{aligned}
$$

Now, $\mathbf{D}_{\mathbf{x}}^{\otimes k} \psi(\mathbf{x})$ can be obtained by taking $k^{t h}$-order K-derivative of Equation (25) as under:

$$
\begin{array}{r}
\psi^{(k)}(\mathbf{x})=\mathbf{D}_{\mathbf{x}}^{\otimes k} \psi(\mathbf{x})=\frac{1}{(2 \pi)^{d}} \int_{\mathbb{R}^{d}}(\boldsymbol{\lambda})^{\otimes k} \exp \left(\sum_{k=1}^{\infty} \frac{\mathbf{c}_{r}(2 k, d)^{\prime}}{2 k !}(i \boldsymbol{\lambda})^{\otimes 2 k}\right) \\
\cos \left(\mathbf{x}^{\prime} \boldsymbol{\lambda}+\sum_{k=1}^{\infty} \frac{\mathbf{c}_{r}(2 k-1, d)^{\prime}}{(2 k-1) !}(i)^{2 k}(\boldsymbol{\lambda})^{\otimes(2 k-1)}+\frac{k \pi}{2}\right) d \boldsymbol{\lambda}
\end{array}
$$

The above Equation (51) with the previous results on expansions in Equation (49) and Equation (50) can be used to simplify the Equation (48). This derives the Generalized Gram-Charlier (GGC) series expressing an unknown $p d f f(\mathbf{x})$ in terms of the cumulant difference vectors $(\boldsymbol{\delta}(k, d))$ and derivatives of a reference $p d f \psi^{(k)}(\mathbf{x})$ as under:

$$
f(\mathbf{x})=\sum_{k=0}^{\infty}(-1)^{k} \frac{\boldsymbol{\alpha}(k, d)^{\prime}}{k !} \psi^{(k)}(\mathbf{x})
$$


where,

$$
\begin{aligned}
\boldsymbol{\alpha}(0, d)= & 1 \\
\boldsymbol{\alpha}(1, d)= & \boldsymbol{\delta}(1, d) \\
\boldsymbol{\alpha}(2, d)= & \boldsymbol{\delta}(2, d)+\boldsymbol{\delta}(1, d)^{\otimes 2} \\
\boldsymbol{\alpha}(3, d)= & \boldsymbol{\delta}(3, d)+3 \boldsymbol{\delta}(2, d) \otimes \boldsymbol{\delta}(1, d)+\boldsymbol{\delta}(1, d)^{\otimes 3} \\
\boldsymbol{\alpha}(4, d)= & \boldsymbol{\delta}(4, d)+4 \boldsymbol{\delta}(3, d) \otimes \boldsymbol{\delta}(1, d)+3 \boldsymbol{\delta}(2, d)^{\otimes 2}+6 \boldsymbol{\delta}(2, d) \\
\otimes & \boldsymbol{\delta}(1, d)^{\otimes 2}+\boldsymbol{\delta}(1, d)^{\otimes 4} \\
\boldsymbol{\alpha}(5, d)= & \boldsymbol{\delta}(5, d)+5 \boldsymbol{\delta}(4, d) \otimes \boldsymbol{\delta}(1, d)+10 \boldsymbol{\delta}(3, d) \otimes \boldsymbol{\delta}(2, d) \\
& +10 \boldsymbol{\delta}(3, d) \otimes \boldsymbol{\delta}(1, d)^{\otimes 2}+15 \boldsymbol{\delta}(2, d)^{\otimes 2} \otimes \boldsymbol{\delta}(1, d) \\
+ & 10 \boldsymbol{\delta}(2, d) \otimes \boldsymbol{\delta}(1, d)^{\otimes 3}+\boldsymbol{\delta}(1, d)^{\otimes 5} \\
\boldsymbol{\alpha}(6, d)= & \boldsymbol{\delta}(6, d)+6 \boldsymbol{\delta}(5, d) \otimes \boldsymbol{\delta}(1, d)+15 \boldsymbol{\delta}(4, d) \otimes \boldsymbol{\delta}(2, d) \\
& +15 \boldsymbol{\delta}(4, d) \otimes \boldsymbol{\delta}(1, d)^{\otimes 2}+10 \boldsymbol{\delta}(3, d)^{\otimes 2}+60 \boldsymbol{\delta}(3, d) \otimes \boldsymbol{\delta}(2, d) \\
& \otimes \boldsymbol{\delta}(1, d)+20 \boldsymbol{\delta}(3, d) \otimes \boldsymbol{\delta}(1, d)^{\otimes 2}+15 \boldsymbol{\delta}(2, d)^{\otimes 3}+45 \boldsymbol{\delta}(2, d)^{\otimes 2} \\
& \otimes \boldsymbol{\delta}(1, d)^{\otimes 2}+15 \boldsymbol{\delta}(2, d) \otimes \boldsymbol{\delta}(1, d)^{\otimes 4}+\boldsymbol{\delta}(1, d)^{\otimes 6}
\end{aligned}
$$

The above set of equations (53) has exact resemblance with that expressing moments in terms of the cumulants in Section 3.1. This must happen, as Equation (52) for GGC expansion with $\delta(\mathbf{x})$ as a reference $p d f$ is matching Equation (12). This matching proves that $\boldsymbol{\alpha}(k, d)$ is related in same way to $\boldsymbol{\delta}(k, d)$, as $\mathbf{m}(k, d)$ to $\mathbf{c}(k, d)$. That is,:

$$
\sum_{k=0}^{\infty} \boldsymbol{\alpha}(k, d)^{\prime} \frac{\boldsymbol{\lambda}^{\otimes k}}{k !}=\exp \left(\sum_{k=1}^{\infty} \boldsymbol{\delta}(k, d)^{\prime} \frac{\boldsymbol{\lambda}^{\otimes k}}{k !}\right)
$$

Further, the $\boldsymbol{\alpha}(k, d)$ in Equation (52) recursively can be obtained in terms of the cumulant difference vector $\boldsymbol{\delta}(k, d)$ as under:

$$
\boldsymbol{\alpha}(k, d)=\sum_{p=0}^{k-1}\left(\begin{array}{c}
k-1 \\
p
\end{array}\right) \mathbf{K}_{\mathfrak{p} h \leftrightarrow l}^{-1} \boldsymbol{\delta}(k-p, d) \otimes \boldsymbol{\alpha}(p, d)
$$

where, $\mathbf{K}_{\mathfrak{p} h \leftrightarrow l}^{-1}$ is a specific commutation matrix; as described previously; to change the order of the cumulants for Kronecker product such that the expression has decreasing order cumulants from left to the right.

The verification of the derived GGC can be obtained by taking Gaussian density as a reference $p d f$. With Gaussian density as a reference, $\boldsymbol{\delta}(1, d)=\mathbf{0}$ and $\boldsymbol{\delta}(2, d)=\mathbf{0}$. So, the coefficients $\boldsymbol{\alpha}(k, d)$ in Equation $(52)$ can be derived as 
under:

$$
\begin{aligned}
& \boldsymbol{\alpha}(0, d)=1 \\
& \boldsymbol{\alpha}(1, d)=\mathbf{0} \\
& \boldsymbol{\alpha}(2, d)=\mathbf{0} \\
& \boldsymbol{\alpha}(3, d)=\boldsymbol{\delta}(3, d)=\mathbf{c}(3, d) \\
& \boldsymbol{\alpha}(4, d)=\boldsymbol{\delta}(4, d)=\mathbf{c}(4, d) \\
& \boldsymbol{\alpha}(5, d)=\boldsymbol{\delta}(5, d)=\mathbf{c}(5, d) \\
& \boldsymbol{\alpha}(6, d)=\boldsymbol{\delta}(6, d)+10 \boldsymbol{\delta}(3, d)^{\otimes 2}=\mathbf{c}(6, d)+10 \mathbf{c}(3, d)^{\otimes 2}
\end{aligned}
$$

Thus, the GGC series is derived and verified using known examples.

\section{Characteristic function of an unknown random vector in terms of a reference characteristic function}

The GGC derived as in Equation (52) can be used to give the characteristic function of an unknown $p d f$, in terms of the characteristic function of a reference $p d f$. For that taking Fourier transform (F) of Equation (52), we get:

$$
\begin{aligned}
\mathcal{F}_{\mathbf{x}}(\boldsymbol{\lambda}) & =\sum_{k=0}^{\infty}(-1)^{k} \frac{\boldsymbol{\alpha}(k, d)^{\prime}}{k !} \mathrm{F}\left(\psi^{(k)}(\mathbf{x})\right) \\
\mathcal{F}_{\mathbf{x}}(\boldsymbol{\lambda}) & =\left[\sum_{k=0}^{\infty} \boldsymbol{\alpha}(k, d)^{\prime} \frac{(i \boldsymbol{\lambda})^{\otimes k}}{k !}\right] \mathrm{F}(\psi(\mathbf{x})) \quad(\because \text { differentiation property of } \mathbf{F}) \\
& =\exp \left[\sum_{k=1}^{\infty} \boldsymbol{\delta}(k, d)^{\prime} \frac{(i \boldsymbol{\lambda})^{\otimes k}}{k !}\right] \mathcal{F}_{r}(\mathbf{x}) \quad(\because \text { Equation }(54))
\end{aligned}
$$

where, $\mathcal{F}_{r}$ is the characteristic function of the reference $p d f$.

\section{Compact derivation for the Generalized Gram-Charlier expansion}

The compact derivation of Equation (52) follows as under:

$$
\begin{aligned}
\mathcal{F}_{\mathbf{x}}(\boldsymbol{\lambda})= & \exp \left[\sum_{k=1}^{\infty} \mathbf{c}(k, d)^{\prime} \frac{(i \boldsymbol{\lambda})^{\otimes k}}{k !}\right] \quad(\because \text { definition in Equation }(17)) \\
= & \exp \left[\sum_{k=1}^{\infty} \boldsymbol{\delta}(k, d)^{\prime} \frac{(i \boldsymbol{\lambda})^{\otimes k}}{k !}\right] \exp \left[\sum_{k=1}^{\infty} \mathbf{c}_{r}(k, d) \frac{(i \boldsymbol{\lambda})^{\otimes k}}{k !}\right] \\
& \left(\because \boldsymbol{\delta}(k, d)=\mathbf{c}(k, d)-\mathbf{c}_{r}(k, d)\right) \\
= & {\left[\sum_{k=0}^{\infty} \boldsymbol{\alpha}(k, d)^{\prime} \frac{(i \boldsymbol{\lambda})^{\otimes k}}{k !}\right] \mathrm{F}(\psi(\mathbf{x})) }
\end{aligned}
$$


Taking inverse Fourier transform of the above equation brings

$$
\begin{aligned}
f(\mathbf{x}) & =\sum_{k=0}^{\infty} \boldsymbol{\alpha}(k, d)^{\prime} \frac{(-1)^{k}}{k !} \boldsymbol{\delta}^{(k)}(\mathbf{x}) * \psi(\mathbf{x}) \\
\text { or } f(\mathbf{x}) & =\sum_{k=0}^{\infty} \boldsymbol{\alpha}(k, d)^{\prime} \frac{(-1)^{k}}{k !} \psi^{(k)}(\mathbf{x})
\end{aligned}
$$

where, * indicates convolution. Thus, the Equation (52) is obtained in a more compact way.

\section{Conclusion}

The article has derived multivariate Generalized Gram-Charlier (GGC) expansion in Equation (52); combined with Equation (55); that expresses an unknown multivariate $p d f$ in terms of vector cumulants and vector derivatives of a reference $p d f$. The multivariate Gram-Charlier A series is derived in Equation (45) and Equation (46) representing an unknown multivariate $p d f$ in terms of its vector cumulants and vector Hermite polynomials. There has been also derived compact formulas for obtaining multivariate vector moments from vector cumulants in Equation (21) and vise-a-verse in Equation (22); the integral form of multivariate $p d f$ representation in Equation (25) and the integral form of multivariate vector Hermite polynomials in Equation (33), as well, in Equation (34). The expressions are derived using only elementary calculus of several variables in vector notations through Kronecker product based derivative operator. Thus, they are more transparent and more comprehensive compare to their corresponding multi-linear matrix representations or tensor representations.

\section{Appendix A: The Multivariate Representations of GCA Series and GGC Series}

As mention in Section 1, this section of the appendix describes existing representations of GCA series and GGC series. The goal is to place together various historical representations for the ease of comparison, on the level of difficulty or simplicity in representation, to the readers. Therefore, no attempt is made to explain their derivation or each terms in representation. For further details the actual references need be referred.

The GCA series representation using multi-element matrix notations for cumulants and moments by Sauer and Heydt (1979) is as under:

$$
\begin{aligned}
f_{\mathbf{x}}(\mathbf{x})= & \sum_{s_{1}=0}^{\infty} \sum_{s_{2}=0}^{\infty} \ldots \sum_{s_{d}=0}^{\infty}\left[\mathbf{C}_{s_{1} s_{2} \cdots s_{d}} \cdot(-1)^{\sum_{i=1}^{d} s_{i}} \prod_{p=1}^{d} H_{s_{p}}\left(x_{p}\right) G\left(x_{p}\right)\right] \\
& \text { with, } \mathbf{C}_{s_{1} s_{2} \cdots s_{d}}=\frac{E\left\{\prod_{i=1}^{d} H_{s_{i}}\left(X_{i}\right)\right\}}{(-1)^{\sum_{i=1}^{d} s_{i}} \prod_{j=1}^{d} s_{j} !}
\end{aligned}
$$


where, $\mathbf{C}_{s_{1} s_{2} \cdots s_{d}}$ is the constant depending upon cross-moments and $H_{i}(x)$ is the one-dimensional Hermite polynomial of $i^{t h}$ order.

The GCA Series representation using recursive formula for Hermite polynomials by Berkowitz and Garner (1970) is as under:

$$
\begin{aligned}
f_{\mathbf{x}}(\mathbf{x})= & G(\mathbf{x}) \sum_{m=0}^{\infty} A_{m} H_{m}(\mathbf{z}) \\
& \text { with, } A_{m}=\prod_{i=1}^{d}\left(m_{i} !\right)^{-1} \int_{\mathbb{R}} J_{m}(z) f_{\mathbf{x}}(\mathbf{x}) d \mathbf{x}
\end{aligned}
$$

where, $G(\mathbf{x})$ denote the multivariate Gaussian; $\mathbf{z}=(\mathbf{x}-\boldsymbol{\mu})$ is the normalized variable; $\boldsymbol{\mu}$ is the mean vector; $\left\{H_{m}(\mathbf{x})\right\}$ and $\left\{J_{m}(\mathbf{x})\right\}$ are the complete biorthogonal system of Hermite polynomials.

Using recursive relations of $\left\{J_{m}(\mathbf{x})\right\}, A_{m}$ is given recursively as under:

$$
A_{m}=\frac{1}{N}\left[\prod_{i=1}^{d}\left(m_{i} !\right)^{-1} \sum_{i=1}^{N} z_{k}^{(i)} J_{m-e_{k}}\left(y^{i}\right)-\sum_{i=1}^{N} r_{k i} m_{k}^{-1} A_{m-e_{k}-e_{f}}\right], k=1, \ldots, d
$$

where, $\mathrm{N}$ is the number of available samples and $\mathbf{e}_{k}$ is a vector with a "1" as the $k^{t h}$ component and " 0 " elsewhere. This defines the coefficients of expansions also recursively.

The GGC series representation using tensor notations for cumulants and Hermite polynomials by McCullagh (1987, Chapter 5) is as under:

$$
f_{\mathbf{x}}(x ; \kappa)=f_{0}(\mathbf{x})\left[1+\eta^{i} h_{i}(\mathbf{x})+\eta^{i j} h_{i j}(\mathbf{x}) / 2 !+\eta^{i j k} h_{i j k}(\mathbf{x}) / 3 !+\ldots\right]
$$

where, $h_{i}(\mathbf{x})=h_{i}(\mathbf{x} ; \boldsymbol{\lambda})=f_{i}(\mathbf{x}) / f_{0}(\mathbf{x}), \quad h_{i j}(\mathbf{x})=h_{i j}(\mathbf{x} ; \boldsymbol{\lambda})=f_{i j}(\mathbf{x}) / f_{0}(\mathbf{x}), \ldots$ and $f_{i}(\mathbf{x})=\partial f_{0}(\mathbf{x}) / \partial x^{i}, f_{i j}(\mathbf{x})=\partial^{2} f_{0}(\mathbf{x}) / \partial x^{i} \partial x^{j}, \ldots ;$ so on. Also, given

$\kappa^{i}, \kappa^{i, j}, \kappa^{i, j, k}, \ldots$ are the cumulant tensors of random vector $\mathbf{x}$ and $\lambda^{i}, \lambda^{i, j}, \lambda^{i, j, k}, \ldots$ are the cumulant tensors of the reference $p d f f_{0}(\mathbf{x})$; we get:

$$
\eta^{i}=\kappa^{i}-\lambda^{i}, \eta^{i, j}=\kappa^{i, j}-\lambda^{i, j}, \eta^{i, j, k}=\kappa^{i, j, k}-\lambda^{i, j, k}, \ldots
$$

The formal 'moments' $\eta^{i}, \eta^{i j}, \eta^{i j k}, \ldots$ are defined based on the formal 'cumulants' (or the cumulant differences) $\eta^{i}, \eta^{i, j}, \eta^{i, j, k}$ and so on.

Taking $f_{0}(\mathbf{x})=G(\mathbf{x})$ i.e. multivariate Gaussian density as the reference $p d f$ and taking $\eta^{i}=0, \eta^{i, j}=0$ in above Equation (70); the GCA series based on cumulant tensors is written as under:

$$
\begin{aligned}
f_{\mathbf{x}}(x ; \kappa)= & G(\mathbf{x})\left[1+\kappa^{i, j, k} h_{i j k}(\mathbf{x}) / 3 !+\kappa^{i, j, k, l} h_{i j k l}(\mathbf{x}) / 4 !+\kappa^{i, j, k, l, m} h_{i j k l m}(\mathbf{x}) / 5 !\right. \\
& \left.+\left(\kappa^{i, j, k, l, m, n}+10 \kappa^{i, j, k} \kappa^{l, m, n}\right) h_{i j k l m n}(\mathbf{x}) / 6 ! \ldots\right]
\end{aligned}
$$

As could be observed, the GGC series and GCA series using tensor notations adds quite an ease to representation. But, with increase in number of terms, the difficulty in representation increases. 
The GCA series using vector moments and vector Hermite polynomials by Holmquist (1996) is as under:

$$
f_{\mathbf{x}}(\mathbf{x})=G\left(\mathbf{x}-\boldsymbol{\mu} ; \mathbf{C}_{\mathbf{x}}\right) \sum_{k=0}^{\infty} \frac{1}{k !} G_{k}^{T}\left(\mathbf{x}-\boldsymbol{\mu} ; \mathbf{C}_{\mathbf{x}}\right) E\left\{\mathbf{H}_{k}\left(\mathbf{x}-\boldsymbol{\mu} ; \mathbf{C}_{\mathbf{x}}\right)\right\}
$$

where, $G_{k}(\mathbf{x}-\boldsymbol{\mu})$ is the $k^{t h}$ order vector derivative of $G(\mathbf{x}-\boldsymbol{\mu})$ and $E\left\{\mathbf{H}_{k}(\mathbf{x}-\boldsymbol{\mu})\right\}$ is the expectation of $k^{\text {th }}$ order vector Hermite polynomial that is the function of vector moments.

$$
E_{f}\left\{\mathbf{H}_{k}\left(\mathbf{X}-\boldsymbol{\mu} ; \mathbf{C}_{\mathbf{x}}^{-1}\right)^{\otimes j}\right\}=k ! \mathbf{S}_{d 1_{k}} \sum_{j=0}^{[k / 2]} \frac{\mathbf{m}_{k-2 j} \otimes\left(-V e c \mathbf{C}_{\mathbf{x}}\right)^{\otimes j}}{(k-2 j) ! j ! 2^{j}}
$$

where, $E_{f}\left\{(\mathbf{X}-\boldsymbol{\mu})^{\otimes j}\right\} \equiv \mathbf{m}_{j}(\boldsymbol{\mu})$.

The GCA series representation using Bell polynomials is obtained by Withers and Nadarajah (2014). Here, the Bell polynomials are represented through cumulant tensors. With $r=\left(r_{1}, r_{2}, \ldots, r_{d}\right) \in \mathbb{I}^{d}, \mathbf{x}^{r}=x_{1}^{r_{1}} \ldots x_{d}^{r_{d}}, r !=r_{1} ! r_{2} ! \ldots r_{d} !$ and $|r|=r_{1}+r_{2}+\ldots+r_{d}$; the GCA series is as under:

$$
\begin{aligned}
f_{\mathbf{x}}(\mathbf{x}) / G(\mathbf{x})-1 & =\sum_{|r| \geq 3}^{\infty} \mathbf{B}_{r} H_{r}\left(x, \mathbf{C}_{\mathbf{x}}\right) / r ! \\
\text { where, } \mathbf{B}_{r} & =\sum_{j=0}^{|r|} \mathbf{B}_{r, j} \\
\mathbf{B}_{r, j} & =\frac{1}{k_{1}(r-j)} \sum_{l=1}^{r-j}\left(\begin{array}{c}
r \\
j
\end{array}\right)\left(j+1-\frac{r+1}{l+1}\right) k_{l+1} \mathbf{B}_{r-l, j} \\
k_{r} & =\kappa\left(X_{r 1}, X_{r 2}, \ldots, X_{r d}\right)
\end{aligned}
$$

where, $k_{r}$ is the $r^{t h}$ order cumulant and $\kappa(\mathbf{x})$ is the moment.

Finally, the existing representations described in this Section A can be compared with those derived in the current article. , can be compared with the GCA series derived in Equation (45) and the GGC series derived in Equation (52) combined with Equation (54). More specifically, the GCA series in Equation (65) using multi-element array representation and the GGC series in Equation (70) using tensor representation can be compared with the GCA series derived in Equation (45) and the GGC series derived in Equation (52), combined with Equation (54), in vector notations. The comparison demonstrates the ease obtained in representation. The same advantages are also obtained for other intermediate results in the article.

\section{Appendix B: Some important properties of the K-derivative operator}

Some important properties of the K-derivative operator are listed below. 
Property 1 (Scaling Property). Let $\boldsymbol{\lambda}=\left(\lambda_{1}, \lambda_{2}, \ldots, \lambda_{d}\right)^{\prime}, \boldsymbol{\lambda} \in \mathbb{R}^{d}, \mathbf{f}(\boldsymbol{\lambda}) \in \mathbb{R}^{m}$ and $\mathbf{f}_{1}(\boldsymbol{\lambda})=\mathbf{A f}(\boldsymbol{\lambda})$, where $\mathbf{A}$ is an $n \times m$ matrix. Then

$$
\mathbf{D}_{\lambda}^{\otimes}\left(\mathbf{f}_{1}\right)=\left(\mathbf{A} \otimes \mathbf{I}_{d}\right) \mathbf{D}_{\lambda}^{\otimes}(\mathbf{f})
$$

where, $\mathbf{I}_{d}$ is a d-dimensional unit matrix.

Property 2 (Chain Rule). Let $\boldsymbol{\lambda}=\left(\lambda_{1}, \lambda_{2}, \ldots, \lambda_{d}\right)^{\prime}, \boldsymbol{\lambda} \in \mathbb{R}^{d}, \mathbf{f}(\boldsymbol{\lambda}) \in \mathbb{R}^{m_{1}}$ and $\mathrm{g}(\boldsymbol{\lambda}) \in \mathbb{R}^{m_{2}}$. Then

$$
\mathbf{D}_{\lambda}^{\otimes}(\mathbf{f} \otimes \mathbf{g})=\mathbf{K}_{3 \leftrightarrow 2}^{-1}\left(m_{1}, m_{2}, d\right)\left(\left(\mathbf{D}_{\lambda}^{\otimes} \mathbf{f}\right) \otimes \mathbf{g}\right)+\mathbf{f} \otimes\left(\mathbf{D}_{\lambda}^{\otimes} \mathbf{g}\right)
$$

where, $\mathbf{K}_{3 \leftrightarrow 2}\left(m_{1}, m_{2}, d\right)$ is a commutation matrix of size $m_{1} m_{2} d \times m_{1} m_{2} d$ that changes the order of the the Kronecker product components. For example,

$$
\mathbf{K}_{3 \leftrightarrow 2}\left(m_{1}, m_{2}, d\right)\left(\mathbf{a}_{\mathbf{1}} \otimes \mathbf{a}_{\mathbf{2}} \otimes \mathbf{a}_{\mathbf{3}}\right)=\mathbf{a}_{\mathbf{1}} \otimes \mathbf{a}_{\mathbf{3}} \otimes \mathbf{a}_{\mathbf{2}}
$$

The above K-derivative properties can be used to derive further the following properties:

1. $\mathbf{D}_{\lambda}^{\otimes} \boldsymbol{\lambda}=V e c \mathbf{I}_{d}$

2. $\mathbf{D}_{\lambda}^{\otimes} \boldsymbol{\lambda}^{\otimes k}=k\left(\boldsymbol{\lambda}^{\otimes k-1} \otimes V e c \mathbf{I}_{d}\right)$

3. Let $g(\boldsymbol{\lambda})=\mathbf{a}^{\prime} f(\boldsymbol{\lambda})$

$\mathbf{D}_{\lambda}^{\otimes} g(\boldsymbol{\lambda})=\left(\mathbf{a} \otimes \mathbf{I}_{d}\right)^{\prime} \mathbf{D}_{\lambda}^{\otimes} f(\boldsymbol{\lambda})$

4. $\mathbf{D}_{\lambda}^{\otimes} \mathbf{a}^{\prime} \boldsymbol{\lambda}^{\otimes k}=k\left(\mathbf{a} \otimes \mathbf{I}_{d}\right)^{\prime}\left(\boldsymbol{\lambda}^{\otimes k-1} \otimes V e c \mathbf{I}_{d}\right)=k \mathbf{a}^{\prime}\left(\boldsymbol{\lambda}^{\otimes k-1} \otimes \mathbf{I}_{d}\right)$

5. $\mathbf{D}_{\boldsymbol{\lambda}}^{\otimes} \mathbf{a}^{\prime \otimes k} \boldsymbol{\lambda}^{\otimes k}=k\left(\mathbf{a}^{\otimes k} \otimes \mathbf{I}_{d}\right)^{\prime}\left(\boldsymbol{\lambda}^{\otimes k-1} \otimes V e c \mathbf{I}_{d}\right)=k\left(\mathbf{a}^{\prime} \boldsymbol{\lambda}\right)^{\otimes k-1} \otimes \mathbf{a}$

6. $\mathbf{D}_{\boldsymbol{\lambda}}^{\otimes r} \boldsymbol{\lambda}^{\otimes k}=k(k-1) \cdots(k-r+1)\left(\boldsymbol{\lambda}^{\otimes k-r} \otimes\left(V e c \mathbf{I}_{d}\right)^{\otimes r}\right)$

( $\because$ Repeated application of the Chain Rule )

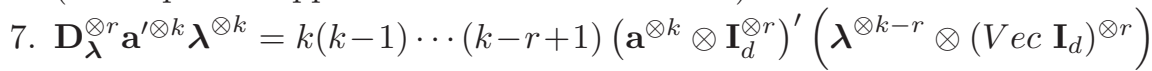

$=k(k-1) \cdots(k-r+1)\left(\mathbf{a}^{\prime} \boldsymbol{\lambda}\right)^{\otimes k-r} \otimes \mathbf{a}^{\otimes r}$

( $\because$ Repeated application of the Chain Rule and the property: ) 


\section{Appendix C: The Taylor series expansion of some required} functions near zero

The Taylor series expansion of the required functions near $\boldsymbol{\lambda}=\mathbf{0}$, based on the Equation (7), are given as under:

$$
\begin{aligned}
\mathrm{e}^{\mathbf{a}^{\prime} \mathbf{x}} & =\sum_{k=0}^{\infty} \frac{\left(\mathbf{a}^{\prime} \mathbf{x}\right)^{\otimes k}}{k !}=1+\mathbf{a}^{\prime} \mathbf{x}+\frac{\left(\mathbf{a}^{\prime} \mathbf{x}\right)^{\otimes 2}}{2 !}+\frac{\left(\mathbf{a}^{\prime} \mathbf{x}\right)^{\otimes 3}}{3 !}+\ldots \\
\sin \left(\mathbf{a}^{\prime} \mathbf{x}\right) & =\sum_{k=0}^{\infty} \frac{(-1)^{k}}{(2 k+1) !}\left(\mathbf{a}^{\prime} \mathbf{x}\right)^{\otimes 2 k+1}=\mathbf{a}^{\prime} \mathbf{x}-\frac{\left(\mathbf{a}^{\prime} \mathbf{x}\right)^{\otimes 3}}{3 !}+\frac{\left(\mathbf{a}^{\prime} \mathbf{x}\right)^{\otimes 5}}{5 !}-\ldots \\
\cos \left(\mathbf{a}^{\prime} \mathbf{x}\right)= & \sum_{k=0}^{\infty} \frac{(-1)^{k}}{(2 k) !}\left(\mathbf{a}^{\prime} \mathbf{x}\right)^{\otimes 2 k}=1-\frac{\left(\mathbf{a}^{\prime} \mathbf{x}\right)^{\otimes 2}}{2 !}+\frac{\left(\mathbf{a}^{\prime} \mathbf{x}\right)^{\otimes 4}}{4 !}-\ldots \\
\mathrm{e}^{\mathbf{a}^{\prime} \mathbf{x}} \cos \left(\mathbf{b}^{\prime} \mathbf{y}\right)= & 1+\mathbf{a}^{\prime} \mathbf{x}+\frac{\left(\mathbf{a}^{\prime} \mathbf{x}\right)^{\otimes 2}}{2 !}-\frac{\left(\mathbf{b}^{\prime} \mathbf{y}\right)^{\otimes 2}}{2 !}+\frac{\left(\mathbf{a}^{\prime} \mathbf{x}\right)^{\otimes 3}}{3 !}-\frac{\left(\mathbf{a}^{\prime} \mathbf{x}\right) \otimes\left(\mathbf{b}^{\prime} \mathbf{y}\right)^{\otimes 2}}{2 !} \\
& +\frac{\left(\mathbf{a}^{\prime} \mathbf{x}\right)^{\otimes 4}}{4 !}+\frac{\left(\mathbf{b}^{\prime} \mathbf{y}\right)^{\otimes 4}}{4 !}-\frac{\left(\mathbf{a}^{\prime} \mathbf{x}\right)^{\otimes 2} \otimes\left(\mathbf{b}^{\prime} \mathbf{y}\right)^{\otimes 2}}{2 ! 2 !}+\ldots \\
\mathrm{e}^{\mathbf{a}^{\prime} \mathbf{x}} \sin \left(\mathbf{b}^{\prime} \mathbf{y}\right)= & \mathbf{b}^{\prime} \mathbf{y}+\left(\mathbf{a}^{\prime} \mathbf{x}\right) \otimes\left(\mathbf{b}^{\prime} \mathbf{y}\right)-\frac{\left(\mathbf{a}^{\prime} \mathbf{x}\right)^{\otimes 2} \otimes\left(\mathbf{b}^{\prime} \mathbf{y}\right)}{2 !}-\frac{\left(\mathbf{b}^{\prime} \mathbf{y}\right)^{\otimes 3}}{3 !} \\
& -\frac{\left(\mathbf{a}^{\prime} \mathbf{x}\right) \otimes\left(\mathbf{b}^{\prime} \mathbf{y}\right)^{\otimes 3}}{3 !}+\frac{\left(\mathbf{b}^{\prime} \mathbf{y}\right)^{\otimes 5}}{5 !}-\frac{\left(\mathbf{a}^{\prime} \mathbf{x}\right)^{\otimes 2} \otimes\left(\mathbf{b}^{\prime} \mathbf{y}\right)^{\otimes 3}}{2 ! 3 !}+\ldots
\end{aligned}
$$

\section{Appendix D: Some Proofs}

\section{D.1. K-derivative of $\delta(\mathrm{x})$}

Based on the Differentiation Property of Fourier Transform, the following is obtained:

$$
\begin{gathered}
\mathrm{F}^{-1}\left(\mathrm{~F}\left(\mathbf{D}^{\otimes k} f(\mathbf{x})\right)=\mathrm{F}^{-1}\left((i \boldsymbol{\lambda})^{\otimes k} \mathrm{~F}(f(\mathbf{x}))\right)\right. \\
\Rightarrow \mathbf{D}^{\otimes k} \delta(\mathbf{x})=\mathrm{F}^{-1}(i \boldsymbol{\lambda})^{\otimes k}
\end{gathered}
$$


D.2. Relationship between $\mathrm{m}(k, d)$ and $\mathrm{c}(k, d)$ for $k=2$ and $k=3$

For $k=2$; using Equation (15), Equation (19) and the differentiation rules for Kronecker product in Appendix B; the following is derived:

$$
\begin{aligned}
& \mathbf{m}(2, d)=\left.\mathbf{D}_{\boldsymbol{\lambda}}^{\otimes 2} \mathbf{M}(\boldsymbol{\lambda})\right|_{\boldsymbol{\lambda}=\mathbf{0}}
\end{aligned}
$$

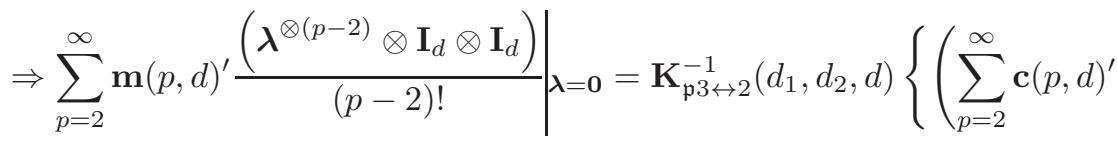

$$
\begin{aligned}
& \left.\left.\frac{\left(\boldsymbol{\lambda}^{\otimes(p-2)} \otimes \mathbf{I}_{d} \otimes \mathbf{I}_{d}\right)}{(p-2) !}\right) \otimes \exp \left(\sum_{q=1}^{\infty} \mathbf{c}(q, d)^{\prime} \frac{\boldsymbol{\lambda}^{\otimes q}}{q !}\right)\right\} \boldsymbol{\lambda}_{\boldsymbol{\lambda}=\mathbf{0}} \\
& +\left\{\left(\sum_{p=1}^{\infty} \mathbf{c}(p, d)^{\prime} \frac{\left(\boldsymbol{\lambda}^{\otimes(p-1)} \otimes \mathbf{I}_{d}\right)}{(p-1) !}\right)^{\otimes 2} \otimes \exp \left(\sum_{q=1}^{\infty} \mathbf{c}(q, d)^{\prime} \frac{\boldsymbol{\lambda}^{\otimes q}}{q !}\right)\right\} \boldsymbol{\lambda}_{\boldsymbol{\lambda}=\mathbf{0}} \\
& \Rightarrow \mathbf{m}(2, d)=\mathbf{c}(2, d)+\mathbf{c}(1, d)^{\otimes 2}
\end{aligned}
$$

where, $\mathfrak{p} 3 \leftrightarrow 2 \in \mathfrak{P}$ is the required permutation and $\mathbf{K}_{\mathfrak{p} 3 \leftrightarrow 2}\left(d_{1}, d_{2}, d\right)$ is the corresponding commutation matrix.

For $k=3$; using Equation (15), Equation (19) and the differentiation rules for 
Kronecker product in Appendix B; the following is derived:

$$
\begin{aligned}
& \mathbf{m}(3, d)=\left.\mathbf{D}_{\boldsymbol{\lambda}}^{\otimes 3} \mathbf{M}(\boldsymbol{\lambda})\right|_{\boldsymbol{\lambda}=\mathbf{0}}
\end{aligned}
$$

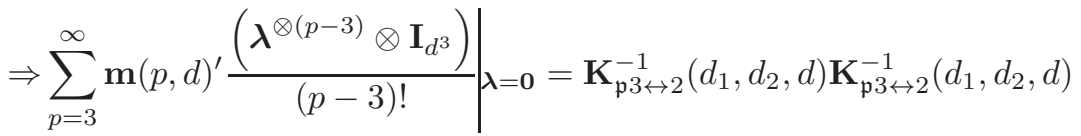

$$
\begin{aligned}
& \left.\left\{\left(\sum_{p=3}^{\infty} \mathbf{c}(p, d)^{\prime} \frac{\left(\boldsymbol{\lambda}^{\otimes(p-3)} \otimes \mathbf{I}_{d^{3}}\right)}{(p-3) !}\right) \otimes \exp \left(\sum_{q=1}^{\infty} \mathbf{c}(q, d)^{\prime} \frac{\boldsymbol{\lambda}^{\otimes q}}{q !}\right)\right\}\right|_{\boldsymbol{\lambda}=\mathbf{0}} \\
& +\mathbf{K}_{\mathfrak{p} 3 \leftrightarrow 2}^{-1}\left(d_{1}, d_{2}, d\right)\left\{( \sum _ { p = 2 } ^ { \infty } \mathbf { c } ( p , d ) ^ { \prime } \frac { ( \boldsymbol { \lambda } ^ { \otimes ( p - 2 ) } \otimes \mathbf { I } _ { d ^ { 2 } } ) } { ( p - 2 ) ! } ) \otimes \left(\sum_{p=1}^{\infty} \mathbf{c}(p, d)^{\prime}\right.\right. \\
& \left.\left.\frac{\left(\boldsymbol{\lambda}^{\otimes(p-1)} \otimes \mathbf{I}_{d}\right)}{(p-1) !}\right) \otimes \exp \left(\sum_{q=1}^{\infty} \mathbf{c}(q, d)^{\prime} \frac{\boldsymbol{\lambda}^{\otimes q}}{q !}\right)\right\} \mid \boldsymbol{\lambda = 0}+\mathbf{K}_{\mathfrak{p} 3 \leftrightarrow 2}^{-1}\left(d_{1}, d_{2}, d\right) \\
& \left\{2\left(\sum_{p=2}^{\infty} \mathbf{c}(p, d)^{\prime} \frac{\left(\boldsymbol{\lambda}^{\otimes(p-2)} \otimes \mathbf{I}_{d^{2}}\right)}{(p-2) !}\right)^{\otimes 1} \otimes\left(\sum_{p=1}^{\infty} \mathbf{c}(p, d)^{\prime} \frac{\left(\boldsymbol{\lambda}^{\otimes(p-1)} \otimes \mathbf{I}_{d}\right)}{(p-1) !}\right)^{\otimes 1}\right. \\
& \left.\otimes \exp \left(\sum_{q=1}^{\infty} \mathbf{c}(q, d)^{\prime} \frac{\boldsymbol{\lambda}^{\otimes q}}{q !}\right)\right\}\left.\right|_{\boldsymbol{\lambda}=\mathbf{0}}+\left(\sum_{p=1}^{\infty} \mathbf{c}(p, d)^{\prime} \frac{\left(\boldsymbol{\lambda}^{\otimes(p-1)} \otimes \mathbf{I}_{d}\right)}{(p-1) !}\right)^{\otimes 2} \otimes\left(\sum_{p=1}^{\infty} \mathbf{c}(p, d)^{\prime}\right. \\
& \left.\frac{\left(\boldsymbol{\lambda}^{\otimes(p-1)} \otimes \mathbf{I}_{d}\right)}{(p-1) !}\right)\left.^{\otimes 1} \exp \left(\sum_{q=1}^{\infty} \mathbf{c}(q, d)^{\prime} \frac{\boldsymbol{\lambda}^{\otimes q}}{q !}\right)\right|_{\boldsymbol{\lambda}=\mathbf{0}} \\
& \Rightarrow \mathbf{m}(3, d)=\mathbf{c}(3, d)+3 \mathbf{c}(2, d) \otimes \mathbf{c}(1, d)+\mathbf{c}(1, d)^{\otimes 3}
\end{aligned}
$$

D.3. Multivariate Gaussian representation in terms of the cumulants (The proof related to the Section 4

Applying $\mathbf{D}_{\mathbf{x}}^{\otimes}$ on Equation (28),

$$
\begin{aligned}
& \mathbf{D}_{\mathbf{x}}^{\otimes} f(\mathbf{x})=\frac{1}{(2 \pi)^{d}} \int_{\mathbb{R}^{d}}\left(\boldsymbol{\lambda} \otimes \mathbf{I}_{d}\right) \otimes \exp \left(-\frac{\mathbf{c}(2, d)^{\prime}}{2} \boldsymbol{\lambda}^{\otimes 2}\right) \sin \left((\mathbf{x}-\mathbf{c}(1, d))^{\prime} \boldsymbol{\lambda}\right) d \boldsymbol{\lambda} \\
& \text { Let, } U(\boldsymbol{\lambda})=\exp \left(-\frac{\mathbf{c}(2, d)^{\prime}}{2} \boldsymbol{\lambda}^{\otimes 2}\right) \\
& \Rightarrow \mathbf{D}_{\boldsymbol{\lambda}}^{\otimes} U(\boldsymbol{\lambda})=-\mathbf{c}(2, d)^{\prime}\left(\boldsymbol{\lambda} \otimes \mathbf{I}_{d}\right) \exp \left(-\frac{\mathbf{c}(2, d)^{\prime}}{2} \boldsymbol{\lambda}^{\otimes 2}\right) \\
& \text { Also, let } V(\boldsymbol{\lambda})=(\mathbf{x}-\mathbf{c}(1, d)) \cos \left((\mathbf{x}-\mathbf{c}(1, d))^{\prime} \boldsymbol{\lambda}\right) \\
& \Rightarrow \int V d \boldsymbol{\lambda}=\sin \left((\mathbf{x}-\mathbf{c}(1, d))^{\prime} \boldsymbol{\lambda}\right)
\end{aligned}
$$


Using above Equation (90), Equation (92) in Equation (89) and taking inv $\mathbf{c}(2, d)=$ $\operatorname{Vec}\left(\mathbf{C}_{\mathbf{x}}^{-1}\right)$; we get:

$$
\mathbf{D}_{\mathbf{x}}^{\otimes} f(\mathbf{x})=-\frac{i n v \mathbf{c}(2, d)^{\prime}}{(2 \pi)^{d}} \int_{\mathbb{R}^{d}}\left(\mathbf{D}_{\boldsymbol{\lambda}}^{\otimes} U(\boldsymbol{\lambda})\right)\left(\int V d \boldsymbol{\lambda}\right) d \boldsymbol{\lambda}
$$

Applying integration by parts to above Equation (94), we get:

$$
\begin{aligned}
\mathbf{D}_{\mathbf{x}}^{\otimes} f(\mathbf{x})= & -\frac{i n v \mathbf{c}(2, d)^{\prime}}{(2 \pi)^{d}}\left\{\left.\exp \left(-\frac{1}{2} \mathbf{c}(2, d)^{\prime} \boldsymbol{\lambda}^{\otimes 2}\right) \sin \left((\mathbf{x}-\mathbf{c}(1, d))^{\prime} \boldsymbol{\lambda}\right)\right|_{\mathbb{R}^{d}}\right. \\
& \left.-\exp \left(-\frac{\mathbf{c}(2, d)^{\prime}}{2} \boldsymbol{\lambda}^{\otimes 2}\right)(\mathbf{x}-\mathbf{c}(1, d)) \cos \left((\mathbf{x}-\mathbf{c}(1, d))^{\prime} \boldsymbol{\lambda}\right) d \boldsymbol{\lambda}\right\} \\
= & i n v \mathbf{c}(2, d)^{\prime}(\mathbf{x}-\mathbf{c}(1, d)) f(\mathbf{x})
\end{aligned}
$$

The solution of above differential equation leads to the following:

$$
\begin{aligned}
f(\mathbf{x}) & =c \exp \left(-(\text { inv } \mathbf{c}(2, d))^{\prime}(\mathbf{x}-\mathbf{c}(1, d))\right) \\
\text { where, } c & =f(\mathbf{0})=\frac{1}{(2 \pi)^{d}} \int_{\mathbb{R}^{d}} \exp \left(-\frac{\mathbf{c}(2, d)^{\prime}}{2} \boldsymbol{\lambda}^{\otimes 2}\right) d \boldsymbol{\lambda} \\
& =(2 \pi)^{-d / 2}\left(\left|\mathbf{C}_{\mathbf{x}}\right|\right)^{-1 / 2} \\
\Rightarrow f(\mathbf{x}) & =(2 \pi)^{-d / 2}\left(\left|\mathbf{C}_{\mathbf{x}}\right|\right)^{-1 / 2} \exp \left(-(\text { inv } \mathbf{c}(2, d))^{\prime}(\mathbf{x}-\mathbf{c}(1, d))\right) \\
\Rightarrow \frac{1}{(2 \pi)^{d}} & \int_{\mathbb{R}^{d}} \exp \left(-\frac{\mathbf{c}(2, d)^{\prime}}{2} \boldsymbol{\lambda}^{\otimes 2}\right) \cos \left(\mathbf{x}^{\prime} \boldsymbol{\lambda}-\mathbf{c}(1, d)^{\prime} \boldsymbol{\lambda}\right) d \boldsymbol{\lambda}=G(\mathbf{x})
\end{aligned}
$$

\section{References}

Amari, S.-i., Cichocki, A., Yang, H. H., et al., 1996. A new learning algorithm for blind signal separation. Advances in neural information processing systems, $757-763$.

Amari, S.-i., Kumon, M., 1983. Differential geometry of edgeworth expansions in curved exponential family. Annals Of The Institute Of Statistical Mathematics 35 (1), 1-24.

Aroian, L. A., 12 1937. The type b gram-charlier series. Ann. Math. Statist. $8(4), 183-192$.

Aroian, L. A., 1947. The probability function of the product of two normally distributed variables. The Annals of Mathematical Statistics 18 (2), pp. 265271.

Berberan-Santos, M., Oct. 2007. Expressing a probability density function in terms of another pdf: A generalized gram-charlier expansion. Journal of Mathematical Chemistry 42 (3), 585-594.

Berkowitz, S., Garner, F., 1970. The calculation of multidimensional hermite polynomials and gram-charlier coefficients. mathematics of computation, 537545. 
Boscolo, R., Roychowdhury, V. P., Apr. 2003. On the uniqueness of the minimum of the information-theoretic cost function for the separation of mixtures of nearly gaussian signals. Nara, Japan, pp. 137-141.

Bowers, N. L., 1966. Expansion of probability density functions as a sum of gamma densities with applications in risk theory. Trans. Soc. Actuaries $18(52), 125$.

Cohen, L., Oct. 1998. Generalization of the gram-charlier/edgeworth series andapplication to time-frequency analysis. Multidimensional Syst. Signal Process. 9 (4), 363-372.

Cohen, L., Mar. 2011. On the generalization of the edgeworth/gram-charlier series. Journal of Mathematical Chemistry 49 (3), 625-628.

Davis, A. W., Dec., 1976. Statistical distributions in univariate and multivariate edgeworth populations. Biometrika 63 (3), 661-670.

Del Brio, E. B., Níguez, T.-M., Perote, J., 2009. Gram-charlier densities: A multivariate approach. Quantitative Finance 9 (7), 855-868.

Girolami, M., Fyfe, C., 1996. Negentropy and kurtosis as projection pursuit indices provide generalised ica algorithms. In: A. C, Back A (eds.), NIPS-96 Blind Signal Separation Workshop. Vol. 8.

Hald, A., 2000. The early history of the cumulants and the gram-charlier series. International Statistical Review 68 (2), 137-153.

Hald, A., Steffensen, J., 2002. On the history of series expansions of frequency functions and sampling distributions, 1873-1944. Det Kongelige Danske Videnskabernes Selskab.

Holmquist, B., 1996. The d-variate vector hermite polynomial of order k. Linear Algebra and its Applications 237-238 (0), 155-190, linear Algebra and Statistics: In Celebration of C. R. Rao's 75th Birthday (September 10, 1995).

Hyvärinen, A., Karhunen, J., Oja, E., 2001. Independent Component Analysis. John Wiley, New York, 481+xxii pages.

Jondeau, E., Rockinger, M., 2001. Gram-charlier densities. Journal of Economic Dynamics and Control 25 (10), 1457-1483.

Kollo, T., von Rosen, D., 1998. A unified approach to the approximation of multivariate densities. Scandinavian Journal of Statistics 25 (1), pp. 93-109.

Magnus, J. R., 2010. On the concept of matrix derivative. Journal of multivariate analysis 101 (101), 2200-2206.

Magnus, J. R., Neudecker, H., 1999. Matrix differential calculus with applications in statistics and econometrics. J. Wiley \& Sons, Chichester, New York, Weinheim.

McCullagh, P., 1987. Tensor Methods in Statistics. London; New York: Chapman \& Hall.

Rietz, H. L., 1927. Mathematical Statistics, 1st Edition. Vol. 3 of Carus Mathematical Monographs. Mathematical Association of America.

S. Rao Jammalamadaka, T. S. R., Terdik, G., May, 2006. Higher order cumulants of random vectors and applications to statistical inference and time series. Sankhya: The Indian Journal of Statistics (2003-2007) 68 (2), 326-356.

Sauer, P., Heydt, G., 1979. A convenient multivariate gram-charlier type a series. IEEE Transactions on Communications, 247-248. 
Schleher, D. C., 1977. Generalized gram-charlier series with application to the sum of log-normal variates (corresp.). IEEE Transactions on Information Theory $23(2), 275-280$.

Skovgaard, I. M., 1986. On multivariate edgeworth expansions. International Statistical Review / Revue Internationale de Statistique 54 (2), pp. 169-186.

Terdik, G., 2002. Higher order statistics and multivariate vector hermite polynomials. Teor. Imovir. Mat. Stat. 66, 147-168.

Viehland, L. A., 1994. Velocity distribution functions and transport coefficients of atomic ions in atomic gases by a gramcharlier approach. Chemical Physics 179 (1), $71-92$.

Withers, C. S., Nadarajah, S., 2014. The dual multivariate charlier and edgeworth expansions. Statistics \& Probability Letters 87, 76-85. 